\title{
HYPERBOLICITY ANALYSIS OF POLYDISPERSE SEDIMENTATION MODELS VIA A SECULAR EQUATION FOR THE FLUX JACOBIAN
}

\author{
RAIMUND BÜRGER ${ }^{\mathrm{A}}$, ROSA DONAT ${ }^{\mathrm{B}}$, PEP MULET $^{\mathrm{B}}$, AND CARLOS A. VEGA ${ }^{\mathrm{C}}$
}

\begin{abstract}
Polydisperse suspensions consist of small particles which are dispersed in a viscous fluid, and which belong to a finite number $N$ of species that differ in size or density. Spatially one-dimensional kinematic models for the sedimentation of such mixtures are given by systems of $N$ non-linear first-order conservation laws for the vector $\Phi$ of the $N$ local solids volume fractions of each species. The problem of hyperbolicity of this system is considered here for the models due to Masliyah, Lockett and Bassoon, Batchelor and Wen and Höfler and Schwarzer. In each of these models, the flux vector depends only on a small number $m<N$ of independent scalar functions of $\Phi$, so its Jacobian is a rank- $m$ perturbation of a diagonal matrix. This allows to identify its eigenvalues as the zeros of a particular rational function $R(\lambda)$, which in turn is the determinant of a certain $m \times m$ matrix. The coefficients of $R(\lambda)$ follow from a representation formula due to Anderson [Lin. Alg. Appl. 246:49-70, 1996]. It is demonstrated that the secular equation $R(\lambda)=0$ can be employed to efficiently localize the eigenvalues of the flux Jacobian, and thereby to identify parameter regions of guaranteed hyperbolicity for each model. Moreover, it provides the characteristic information required by certain numerical schemes to solve the respective systems of conservation laws.
\end{abstract}

\section{INTRODUCTION}

1.1. Scope. Polydisperse suspensions consist of small solid particles dispersed in a viscous fluid, where the particles are assumed to belong to a number $N$ of species that differ in size or density. The sedimentation of such mixtures is frequently described by spatially one-dimensional models. If the particles are small compared with the cross-sectional area of the settling vessel, then the $N$ species can be treated as superimposed continuous phases, where species $i$ is associated with the volume fraction $\phi_{i}$, the phase velocity $v_{i}$, size (diameter) $d_{i}$, and density $\varrho_{i}$, where we assume that $d_{1}=1 \geq d_{2} \geq \cdots \geq d_{N}$ and $d_{i} \neq d_{j}$ or $\varrho_{i} \neq \varrho_{j}$ for $i \neq j$. The continuity equations of the $N$ species are then $\partial_{t} \phi_{i}+\partial_{x}\left(\phi_{i} v_{i}\right)=0, i=1, \ldots, N$, where $t$ is time and $x$ is depth. The velocities $v_{1}, \ldots, v_{N}$ are assumed to be given functions of the vector $\Phi:=\Phi(x, t):=$ $\left(\phi_{1}(x, t), \ldots, \phi_{N}(x, t)\right)^{\mathrm{T}}$ of local concentrations. This yields systems of conservation laws of the type

$$
\partial_{t} \Phi+\partial_{x} \mathbf{f}(\Phi)=0, \quad \mathbf{f}(\Phi)=\left(f_{1}(\Phi), \ldots, f_{N}(\Phi)\right)^{\mathrm{T}}, \quad f_{i}(\Phi)=\phi_{i} v_{i}(\Phi), \quad i=1, \ldots, N .
$$

The one-dimensional model (1.1), where the concentrations are the only unknown flow variables, is called kinematic. We are interested in the hyperbolicity analysis of (1.1) for arbitrary $N$ under the assumption that the velocities $v_{1}, \ldots, v_{N}$ are functions of a small number $m(m \ll N)$ of scalar functions of $\Phi$, i.e.,

$$
v_{i}=v_{i}\left(p_{1}, \ldots, p_{m}\right), \quad p_{l}=p_{l}(\Phi), \quad i=1, \ldots, N, \quad l=1, \ldots, m .
$$

Under the present assumptions, the entries $f_{i j}(\Phi):=\partial f_{i}(\Phi) / \partial \phi_{j}$ of the Jacobian $\mathcal{J}_{\mathbf{f}}(\Phi)$ are given by

$$
f_{i j}=\frac{\partial\left(\phi_{i} v_{i}\right)}{\partial \phi_{j}}=v_{i} \delta_{i j}+\sum_{l=1}^{m} \phi_{i} \frac{\partial v_{i}}{\partial p_{l}} \frac{\partial p_{l}}{\partial \phi_{j}}, \quad i, j=1, \ldots, N
$$

Date: June 9, 2009

${ }^{\mathrm{A}} \mathrm{CI}^{2} \mathrm{MA}$ and Departamento de Ingeniería Matemática, Facultad de Ciencias Físicas y Matemáticas, Universidad de Concepción, Casilla 160-C, Concepción, Chile. E-Mail: rburger@ing-mat.udec.cl.

${ }^{\mathrm{B}}$ Departament de Matemàtica Aplicada, Universitat de València, Av. Dr. Moliner 50, E-46100 Burjassot, Spain. E-Mail: donat@uv.es, mulet@uv.es.

${ }^{\mathrm{C}}$ Departamento de Ingeniería Matemática, Facultad de Ciencias Físicas y Matemáticas, Universidad de Concepción, Casilla 160-C, Concepción, Chile. Permanent address: Departamento de Matemáticas y Estadística, División de Ciencias Básicas, Universidad del Norte, Barranquilla, Colombia. E-Mail: cvega@uninorte.edu.co. 
i.e., $\mathcal{J}_{\mathbf{f}}(\Phi)$ is a rank- $m$ perturbation of a diagonal matrix. Models of this type include those by Masliyah [34] and Lockett and Bassoon [33] ("MLB model"), Batchelor [4] and Batchelor and Wen [6] ("BW model"), Davis and Gecol [21] ("DG model"), and Höfler and Schwarzer [13, 29, 30] ("HS model").

Hyperbolicity is an important property for polydisperse models, since it is often related to the range of validity of the models. However, the analysis of the characteristic polynomial of the Jacobian matrix of the system, in order to determine its eigenvalues, is rarely an easy task. Strict hyperbolicity for any $N$ has only been proven for the MLB model, under certain restrictions (for equal-density particles, see [3, 11]).

In [23], the authors provide a proof of the hyperbolicity of the MLB model for equal-density spheres that does not involve an explicit computation of $\operatorname{det}\left(\mathcal{J}_{\mathbf{f}}(\Phi)-\lambda \mathbf{I}\right)$. It exploits the algebraic structure of the Jacobian matrix, and makes use of the fact that the eigenvalues of a rank- $m$ perturbation of a diagonal matrix can be characterized as the roots of the so-called secular equation [1]. The analysis is based on a a rational function, $R(\lambda)$, that satisfies

$$
\operatorname{det}\left(\mathcal{J}_{\mathbf{f}}(\Phi)-\lambda \mathbf{I}\right)=R(\lambda) \prod_{i=1}^{N}\left(v_{i}-\lambda\right)
$$

for a fixed vector $\Phi$, under appropriate circumstances. For $(1.1), R(\lambda)$ is of the form

$$
R(\lambda)=\sum_{i=1}^{N} \frac{\gamma_{i}}{v_{i}-\lambda},
$$

and its coefficients $\gamma_{i}, i=1, \ldots, N$ can be calculated with acceptable effort for moderate values of $m$. The key result is that if these coefficients are of the same sign, then the existence of $N$ different eigenvalues of $\mathcal{J}_{\mathbf{f}}(\Phi)$ is ensured. Moreover, these eigenvalues can be localized since they interlace with $v_{1}, \ldots, v_{N}$. This is also an important property from the numerical point of view, since no explicit formulas for the eigenvalues are available, and its computation must be always carried out by root finders.

This approach has proven to be more convenient than the explicit computation of $\operatorname{det}\left(\mathcal{J}_{\mathbf{f}}(\Phi)-\lambda \mathbf{I}\right)$ by successive row and column eliminations done for polydisperse models in e.g. in [11, 39] (see [23] for the "secular" approach), or for kinematic traffic flow models in [52] (see [24] for the "secular" approach).

It is the purpose of this paper to employ this calculus to provide a new proof of hyperbolicity for variants of the MLB model, and to derive new hyperbolicity results for the BW and HS models. In particular, we identify conditions on the smallest particle size, the maximum solids concentration and certain model parameters under which these models are strictly hyperbolic for arbitrary $N$. Numerical simulations illustrate the MLB and HS models, and demonstrate how the hyperbolicity analysis provides characteristic information required by numerical schemes.

1.2. Related work. For particles that have the same density, and after suitably rescaling the time variable, the components $f_{i}(\Phi), i=1, \ldots, N$ of the flux vector $\mathbf{f}(\Phi)$ of the MLB model can be stated as

$$
f_{i}(\Phi)=\phi_{i}(1-\phi) V(\phi)\left(d_{i}^{2}-\left(\phi_{1} d_{1}^{2}+\cdots+\phi_{N} d_{N}^{2}\right)\right), \quad i=1, \ldots, N,
$$

where $\phi=\phi_{1}+\cdots+\phi_{N}$ and the so-called hindered settling factor $V(\phi)$ is assumed to satisfy

$$
V(0)=1, \quad V\left(\phi_{\max }\right)=0, \quad V^{\prime}(\phi) \leq 0 \quad \text { for } \phi \in\left[0, \phi_{\max }\right],
$$

where $\phi_{\max }$ is the maximum total solids concentration. We consider vectors $\Phi \in \overline{\mathcal{D}}_{\phi_{\max }}$, where $\overline{\mathcal{D}}_{\phi_{\max }}$ is the closure of the set $\mathcal{D}_{\phi_{\max }}:=\left\{\Phi \in \mathbb{R}^{N}: \phi_{1}>0, \ldots, \phi_{N}>0, \phi:=\phi_{1}+\cdots+\phi_{N}<\phi_{\max }\right\}$.

In [14] it was shown that loss of hyperbolicity, that is, the occurrence of pairs of complex-conjugate eigenvalues of $\mathcal{J}_{\mathfrak{f}}(\Phi)$, is an instability criterion for polydisperse suspensions. For $N=2$ this criterion requires evaluating the discriminant $I_{2}(\Phi):=\left(f_{11}(\Phi)-f_{22}(\Phi)\right)^{2}-4 f_{12}(\Phi) f_{21}(\Phi)$; for vectors $\Phi$ with $I_{2}(\Phi)<0$, the system (1.1) is unstable (elliptic) [5]. In [2,12,14], instability regions for $N=2,3$ and different choices of $\mathbf{f}(\Phi)$ are determined, while in [11] it is proven that for equal-density particles $\left(\varrho_{1}=\cdots=\varrho_{N}\right)$, arbitrary $N$ and $d_{i} \neq d_{j}$ for $i \neq j,(1.1)$ with the MLB flux vector (1.5) is strictly hyperbolic for all $\Phi \in \mathcal{D}_{1}$. The consequences of instability include the formation of blobs and "fingers" in bidisperse sedimentation and the formation of nonhomogeneous sediments [12]. These phenomena have been observed in experiments (e.g., in [46]) under 
the circumstances predicted by the instability criterion. For one-dimensional kinematic models, loss of hyperbolicity sometimes predicts anomalous numerical solutions, for example, heavy and buoyant particles block each other within the vessel [10], and the steady-state sediment composition varies continuously [8].

On the other hand, strict hyperbolicity, and thus stability for equal-density spheres agrees with experimental evidence, since instabilities have only been observed with particles of different densities [46]. Consequently, a sound model should be strictly hyperbolic for equal-density particles, at least if the parameter $d_{N}$ is sufficiently close to one. Thus, there is interest in determining a region of guaranteed hyperbolicity of a given model in dependence of $d_{N}$ and $\phi_{\max }$. This region should be independent of $N$, since only $d_{N}$ can be controlled in real applications, for example by sieving. This work outlines a calculus that provides such a criterion for a number of models. While the results for the MLB model have been obtained by other methods (but at considerably more effort, see [11]), the analysis of the BW and HS models is new. In contrast to the MLB model, within the BW model the hindrance of all species to a given species $i$ is not described by a factor $V=V(\phi)$, but by a factor that depends on $1+\mathbf{s}_{i}^{\mathrm{T}} \Phi$, where $\mathbf{s}_{i}^{\mathrm{T}}=\left(S_{i 1}, \ldots, S_{i N}\right)$ is a vector of non-positive coefficients and $S_{i j}<0$ is a function of the size ratio $d_{j} / d_{i}$. The BW model is valid for dilute suspensions only (i.e., for suspensions of small concentration), and the HS and DG models were both proposed as extensions of the $\mathrm{BW}$ model to the whole range of concentrations from the dilute limit to packed sediments. The BW, HS and DG models are algebraically more complicated than the MLB model, and the results of [11], based on deriving the characteristic polynomial of $\mathcal{J}_{\mathbf{f}}(\Phi)$, are difficult to apply in this case. However, if one employs a cubic polynomial dependence of $S_{i j}$ on $d_{j} / d_{i}$, then the BW, HS and DG models become cases of (1.1) and (1.2) for $m=4$, and a hyperbolicity analysis becomes feasible via the secular equation. Nevertheless, to make this paper concise and to focus on the main ideas, we herein set the coefficient of the cubic term to zero. This assumption is also made a priori in part of the literature [13,29, 30], and is otherwise justified by the observation that this coefficient is usually very small. Consequently, we limit the present analysis to the case $m=3$. In addition, we do not analyze herein the DG model, since previous work for $N=2$ and $N=3$ [14] showed that this model is hyperbolic for fairly narrow size distributions only. In future work, however, we will employ the secular equation to explicitly derive bounds of the hyperbolicity region for the DG model.

Although hyperbolicity in the equal-density case is a criterion that helps to decide whether a given polydisperse sedimentation model is formally sound and generates characteristic information important for the implementation of numerical schemes, we do not attempt to judge which model is preferable or more accurate. Rather, we highlight the relevance of our analysis by mentioning that recent works that employ either the MLB model or the BW, DG or HS model include [26, 35, 37, 45, 49, 50] and [19, 22, 28], respectively.

Clearly, this analysis should be extended to additional sedimentation models (see Section 6). Other multispecies kinematic flow models of the type (1.1), (1.2), which are amenable to a similar hyperbolicity analysis, include multi-class vehicular traffic $[7,15,17,24,48,51,52]$ and the creaming of emulsions [15, 39].

1.3. Outline of this paper. In Section 2 we outline the secular equation and its application to (1.1), (1.2), stating the basic hyperbolicity theorem, the "interlacing property" (i.e., the separation of eigenvalues by the velocities), and the computation of eigenvectors. In Section 3 the MLB, BW and HS models of polydisperse sedimentation are stated. In Section 4, which is at the core of this paper, the secular equation is applied to analyze the hyperbolicity of each of these models. For the MLB model (where $m=2$ ), we first present in Section 4.1 a more compact proof of hyperbolicity for equal-density spheres than in $[3,11]$, and then estimate the hyperbolicity region for particles that differ in density only. Then, in Section 4.3, we present a new analysis of the BW model for equal-density spheres by means of the secular equation, which results in a characterization of the parameter range within which the equations are hyperbolic, and in an upper bound of the total concentration up to which this property can be guaranteed. This bound is fairly small, in accordance with the limitation of the BW model to dilute suspensions. Then, in Section 4.4, we analyze the HS model in a similar manner. It turns out that the HS model is strictly hyperbolic if $d_{N}$ is not too small. Section 5 presents some simulations of the MLB and HS models made by the Roe [32] and KurganovTadmor (KT) [31] schemes to illustrate the sedimentation processes and the use of characteristic information provided by the calculus of the secular equation. Section 6 collects some conclusions of our analysis. 


\section{THE SECULAR EQUATION}

According to (1.3), for a fixed vector $\Phi \in \mathcal{D}_{\phi_{\max }}$ the Jacobian $\mathcal{J}_{\mathbf{f}}=\mathcal{J}_{\mathbf{f}}(\Phi)$ is a matrix of the form

$$
\mathcal{J}_{\mathbf{f}}=\mathbf{D}+\mathbf{B A}^{\mathrm{T}}, \quad \mathbf{D}=\operatorname{diag}\left(v_{1}, \ldots, v_{N}\right), \quad\left\{\begin{array}{l}
\mathbf{B}=\left(B_{i l}\right)=\left(\phi_{i} \partial v_{i} / \partial p_{l}\right), \quad 1 \leq i, j \leq N, \quad 1 \leq l \leq m . \\
\mathbf{A}=\left(A_{j l}\right)=\left(\partial p_{l} / \partial \phi_{j}\right),
\end{array}\right.
$$

As a motivation for the secular equation, we briefly summarize the analysis in [23]. To this end, let us assume that $\lambda$ is an eigenvalue of $\mathcal{J}_{\mathbf{f}}(\Phi)$, with eigenvector $\mathbf{x}$, i.e., $\left(\mathbf{D}+\mathbf{B A}^{\mathrm{T}}\right) \mathbf{x}=\lambda \mathbf{x}$ and such that

$$
\lambda \neq v_{i} \quad \text { for all } i=1, \ldots, N .
$$

Since $(\mathbf{D}-\lambda \mathbf{I})$ is invertible, we can also write $\mathbf{x}+(\mathbf{D}-\lambda \mathbf{I})^{-1} \mathbf{B}\left(\mathbf{A}^{\mathrm{T}} \mathbf{x}\right)=0$, and multiplying this relation by $\mathbf{A}^{\mathrm{T}}$, we get

$$
\mathbf{A}^{\mathrm{T}} \mathbf{x}+\mathbf{A}^{\mathrm{T}}(\mathbf{D}-\lambda \mathbf{I})^{-1} \mathbf{B}\left(\mathbf{A}^{\mathrm{T}} \mathbf{x}\right)=0,
$$

i.e., the vector $\boldsymbol{\xi}=\mathbf{A}^{\mathrm{T}} \mathbf{x} \in \mathbb{R}^{m}$ satisfies $\mathbf{M}_{\lambda} \boldsymbol{\xi}=0$, where

$$
\mathbf{M}_{\lambda}:=\mathbf{I}+\mathbf{A}^{\mathrm{T}}(\mathbf{D}-\lambda \mathbf{I})^{-1} \mathbf{B} .
$$

Clearly, we must have $\boldsymbol{\xi} \neq 0$, since otherwise we would have $\mathbf{x}=0$. Hence, any eigenvalue $\lambda \neq v_{i}$ for all $i$ must be a root of the equation $\operatorname{det} \mathbf{M}_{\lambda}=0$, and we obtain a direct relation between the equation $\operatorname{det} \mathbf{M}_{\lambda}=0$ and the eigenvalues of $\mathcal{J}_{\mathbf{f}}$. The optimal situation is when $R(\lambda)=\operatorname{det} \mathbf{M}_{\lambda}$ has $N$ different real roots, since in this case these must be all the eigenvalues of $\mathcal{J}_{\mathbf{f}}$, which ensures strict hyperbolicity of the system (1.1).

The secular equation

$$
R(\lambda)=\operatorname{det} \mathbf{M}_{\lambda}=0
$$

provides, hence, relevant information on the eigenvalues of $\mathcal{J}_{\mathbf{f}}$. Rather than forming explicitly the matrix $\mathbf{M}_{\lambda}$ and compute its determinant, we use a form of the function $R(\lambda)$ that can be traced back to Anderson [1], obtained after using certain algebraic results concerning eigenvalues of rank- $m$ perturbations of a diagonal matrix.

We introduce now the relevant notation. We denote by $S_{r}^{p}$ the set of all (ordered) subsets of $r$ elements taken from a set of $p$ elements. Assuming that $\mathbf{X}$ is an $m \times N$ matrix, and given the index sets $I:=\left\{i_{1}<\right.$ $\left.\cdots<i_{k}\right\} \in S_{k}^{N}$ and $J:=\left\{j_{1}<\cdots<j_{l}\right\} \in S_{l}^{m}$, we denote by $\mathbf{X}^{I, J}$ the $k \times l$ submatrix of $\mathbf{X}$ given by $\left(\mathbf{X}^{I, J}\right)_{p, q}=X_{i_{p}, j_{q}}$. The following theorem can be found in [1], but we give here the form in [23], which provides the explicit formulas to be used in the applications.

Theorem 2.1 (The secular equation, [1,23]). Assume that $\mathbf{D}$ is a diagonal matrix as given by (2.1) with $v_{i}>v_{j}$ for $i<j$, and that $\mathbf{A}$ and $\mathbf{B}$ have the formats specified in (2.1). Let $\lambda \neq v_{i}$ for $i=1, \ldots, N$. Then $\lambda$ is an eigenvalue of $\mathbf{D}+\mathbf{B} \mathbf{A}^{\mathrm{T}}$ if and only if

$$
R(\lambda):=\operatorname{det} \mathbf{M}_{\lambda}=1+\sum_{i=1}^{N} \frac{\gamma_{i}}{v_{i}-\lambda}=0 .
$$

The coefficients $\gamma_{i}, i=1, \ldots, N$, are given by the following expression:

$$
\gamma_{i}=\sum_{r=1}^{\min (N, m)} \sum_{i \in I \in S_{r}^{N}, J \in S_{r}^{m}} \frac{\operatorname{det} \mathbf{A}^{I, J} \operatorname{det} \mathbf{B}^{I, J}}{\prod_{l \in I, l \neq i}\left(v_{l}-v_{i}\right)} .
$$

The importance of the secular equation is elucidated by the following corollary, which is an extended version of [23, Corollary 1].

Corollary 2.1. With the notation of Theorem 2.1, assume that $\gamma_{i} \cdot \gamma_{j}>0$ for $i, j=1, \ldots, N$. Then $\mathbf{D}+\mathbf{B A}^{\mathrm{T}}$ is diagonalizable with real eigenvalues $\lambda_{1}, \ldots, \lambda_{N}$. If $\gamma_{1}, \ldots, \gamma_{N}<0$, the interlacing property

$$
M_{1}:=v_{N}+\gamma_{1}+\cdots+\gamma_{N}<\lambda_{N}<v_{N}<\lambda_{N-1}<\cdots<\lambda_{1}<v_{1}
$$

holds, while for $\gamma_{1}, \ldots, \gamma_{N}>0$, the following analogous property holds:

$$
v_{N}<\lambda_{N}<v_{N-1}<\lambda_{N-1}<\cdots<v_{1}<\lambda_{1}<M_{2}:=v_{1}+\gamma_{1}+\cdots+\gamma_{N} .
$$


Proof. If $v_{i} \neq v_{j}$ for $i \neq j$ and $\gamma_{1}, \ldots, \gamma_{N}$ have the same sign $s$, then $R(\lambda) \rightarrow(\mp s) \infty$ as $\lambda \rightarrow v_{i}^{ \pm}$, hence $R$ has $N-1$ changes of sign between two consecutive poles. Since $R(\lambda) \rightarrow 1$ for $\lambda \rightarrow \pm \infty$, there is another change of sign to the left of $v_{N}$ if $s=-1$ or to the right of $v_{1}$ if $s=1$. Thus, noting that $M_{1}<v_{N}$ and $M_{2}>v_{1}$ in the respective cases $\gamma_{i}<0$ and $\gamma_{i}>0$, the property of $M_{1}$ being a lower bound and $M_{2}$ being an upper bound for $\lambda_{1}, \ldots, \lambda_{N}$, respectively, is established if we can show that $R\left(M_{1}\right) \geq 0$ and $R\left(M_{2}\right) \geq 0$, respectively. In the case $\gamma_{i}<0$ we have that $M_{1}-v_{i}=v_{N}-v_{i}+\gamma_{1}+\cdots+\gamma_{N} \leq \gamma_{1}+\cdots+\gamma_{N}$ for $i=1, \ldots, N$, which implies that $\gamma_{i} /\left(M_{1}-v_{i}\right) \leq \gamma_{i} /\left(\gamma_{1}+\cdots+\gamma_{N}\right)$ for $i=1, \ldots, N$, and therefore

$$
R\left(M_{1}\right)=1+\sum_{i=1}^{N} \frac{\gamma_{i}}{v_{i}-M_{1}} \geq 1-\sum_{i=1}^{N} \frac{\gamma_{i}}{\gamma_{1}+\cdots+\gamma_{N}}=0 .
$$

The proof of $R\left(M_{2}\right) \geq 0$ is analogous.

As a consequence of Corollary 2.1, strict hyperbolicity of (1.1) follows whenever the coefficients $\gamma_{i}$ of the associated secular equation of the system have a uniform sign. The interlacing property is important for numerical schemes, since the actual eigenvalues may be computed conveniently by a root finder. The bounds for the eigenvalues, i.e. the characteristic speeds of the system, are also important for numerical purposes.

Remark 2.1. The conditions of Corollary 2.1 are sufficient for the strict hyperbolicity of the models, but are far from necessary. A slightly weaker set of hypotheses leading to strict hyperbolicity would be that the coefficients $\gamma_{i}$ either have a definite sign or, if $\gamma_{i}=0$, then $v_{i}$ is not a root of the secular equation, for Theorem 2.1 would then anyway provide $N$ different roots of the characteristic polynomial. The condition $R\left(v_{i}\right) \neq 0$ whenever $\gamma_{i}=0$ is hard to analyze in general, but there is a situation where it can be neatly ensured, given, for instance, that $v_{1}>\cdots>v_{N}$ and $\gamma_{j} \leq 0$ for all $j: \gamma_{i}=0$ implies $\gamma_{j}=0$ for all $j<i$, which yields

$$
R\left(v_{i}\right)=1+\sum_{j=i+1}^{N} \frac{\gamma_{j}}{v_{j}-v_{i}}>0 .
$$

Remark 2.2. In the context of models of polydisperse sedimentation, the situation addressed in Remark 2.1 occurs if we choose $\Phi=\left(\phi_{1}, \ldots, \phi_{N}\right)^{\mathrm{T}}$ such that $\phi_{i}=0$ for $1 \leq i \leq i_{0}$ and $\phi_{i} \in\left(0, \phi_{\max }\right)$ for $i_{0}+1 \leq i \leq N$ for an index $i_{0} \in\{1, \ldots, N\}$. Consequently, suppose that we are able to establish strict hyperbolicity on all of $\mathcal{D}_{\phi_{\max }}$, then this property also holds on certain parts of the boundary of $\mathcal{D}_{\phi_{\max }}$; on the remaining parts of that boundary, the model (1.1) is still hyperbolic, but not necessarily strictly hyperbolic.

Remark 2.3. Anderson [1] mentions that for the case of a self-adjoint rank-1 perturbation of a diagonal matrix, Theorem 2.1 was proved first by Golub [25], who also utilizes the expression "secular equation" [25, p. 327] for the algebraic form of $R(\lambda)$ in that case. Nevertheless, this expression is in fact much older, and appeared at least as early as in the work of Cauchy [18], where the adjective secular has the meaning of "existing or continuing through the centuries".

Finally, another important by-product of this derivation is the possibility of having an explicit expression of the spectral decomposition of the Jacobian matrix of the system, which is also a useful asset in numerical simulations. Assume that $\lambda$ is a root of the secular equation, i.e., $\lambda$ is an eigenvalue of $\mathcal{J}_{\mathbf{f}}$ that satisfies (2.2). Then $\boldsymbol{\xi}=\mathbf{A}^{\mathrm{T}} \mathbf{X}$ is a solution of $\mathbf{M}_{\lambda} \boldsymbol{\xi}=0$. But $\mathbf{M}_{\lambda}$ is an $m \times m$ matrix that can easily be computed. Given two vectors $\mathbf{g}=\left(g_{1}, \ldots, g_{N}\right)^{\mathrm{T}}, \mathbf{h}=\left(h_{1}, \ldots, h_{N}\right)^{\mathrm{T}} \in \mathbb{R}^{N}$, if we use the notation

$$
[\mathbf{g}, \mathbf{h}]:=[\mathbf{g}, \mathbf{h}]_{\lambda}:=\mathbf{g}^{\mathrm{T}}(\mathbf{D}-\lambda \mathbf{I})^{-1} \mathbf{h}=\sum_{k=1}^{N} \frac{g_{k} h_{k}}{v_{k}-\lambda}
$$

and denote by $\mathbf{a}_{1}, \ldots, \mathbf{a}_{m}$ and $\mathbf{b}_{1}, \ldots, \mathbf{b}_{m}$ the columns of $\mathbf{A}$ and $\mathbf{B}$, respectively, then

$$
\mathbf{M}_{\lambda}=\mathbf{I}+\left(\left[\mathbf{a}_{i}, \mathbf{b}_{j}\right]\right)_{1 \leq i, j \leq m} .
$$

Assuming that we can compute a non-zero solution $\boldsymbol{\xi}$ of

$$
\mathbf{M}_{\lambda} \boldsymbol{\xi}=0
$$


we can use the relation $\mathbf{x}+(\mathbf{D}-\lambda \mathbf{I})^{-1} \mathbf{B}\left(\mathbf{A}^{\mathrm{T}} \mathbf{x}\right)=0$ to compute a right eigenvalue of $\mathcal{J}_{\mathbf{f}}$ as

$$
\mathbf{x}=-(\mathbf{D}-\lambda \mathbf{I})^{-1} \mathbf{B} \boldsymbol{\xi} .
$$

The same procedure may be employed to calculate the left eigenvectors of $\mathcal{J}_{\mathbf{f}}$, since they are the right eigenvectors of $\mathcal{J}_{\mathbf{f}}^{\mathrm{T}}=\mathbf{D}+\mathbf{A B}^{\mathrm{T}}$. In other words, the roles of $\mathbf{A}$ and $\mathbf{B}$ and corresponding columns need to be interchanged. This will be further illustrated for the MLB model.

For the case given by (2.1) and assuming that $m<N$, we note that with $\mathbf{A}$ and $\mathbf{B}$ defined in (2.1) we can write

$$
\operatorname{det} \mathbf{A}^{I, J}=\operatorname{det}\left(\frac{\partial p_{J}}{\partial \phi_{I}}\right), \quad \operatorname{det} \mathbf{B}^{I, J}=\operatorname{det}\left(\frac{\partial v_{I}}{\partial p_{J}}\right) \prod_{l \in I} \phi_{l},
$$

where the notation above should be self-explanatory. Then, we can write

$$
\gamma_{i}=\phi_{i} \sum_{r=1}^{m} \gamma_{r, i}, \quad \gamma_{r, i}=\sum_{i \in I \in S_{r}^{N}} \prod_{l \in I, l \neq i} \frac{\phi_{l}}{v_{l}-v_{i}} \sum_{J \in S_{r}^{m}} \operatorname{det}\left(\frac{\partial v_{I}}{\partial p_{J}}\right) \operatorname{det}\left(\frac{\partial p_{J}}{\partial \phi_{I}}\right) .
$$

When $m=1$ or $m=2$, these quantities can be easily computed and the hyperbolicity analysis via the secular equation is much less involved than the study of $\operatorname{det}\left(\mathcal{J}_{\mathbf{f}}(\Phi)-\lambda \mathbf{I}\right)$. For $m=3$ or $m=4$, the computations in the secular equation are more involved, but they can be useful in providing at least partial results concerning hyperbolicity, where the theoretical analysis of $\left.\operatorname{det}\left(\mathcal{J}_{\mathbf{f}}(\Phi)\right)-\lambda \mathbf{I}\right)$ is essentially out of reach.

\section{Kinematic MOdELS OF POLYDisPeRse SEDIMENTATION}

3.1. The Masliyah-Lockett-Bassoon (MLB) model. The MLB model is based on the following explicit equation for the solid-fluid relative velocity $u_{i}:=v_{i}-v_{\mathrm{f}}$ of species $i$, also called slip velocity:

$$
u_{i}=\frac{g d_{i}^{2}\left(\bar{\varrho}_{i}-\bar{\varrho}^{\mathrm{T}} \Phi\right)}{18 \mu_{\mathrm{f}}} V_{i}(\Phi),
$$

where $g$ is the acceleration of gravity, $\varrho_{i}:=\varrho_{i}-\varrho_{\mathrm{f}}$ is the reduced density of particle species $i$, where $\varrho_{i}$ and $\varrho_{\mathrm{f}}$ are the mass densities of particle species $i$ and the fluid, respectively, $\mu_{\mathrm{f}}$ is the viscosity of the fluid, $\bar{\varrho}:=\left(\bar{\varrho}_{1}, \ldots, \bar{\varrho}_{N}\right)^{\mathrm{T}}$, and $V_{i}(\Phi)$ is the hindered settling factor for species $i$. In most previous works, this factor is assumed to be the same for all species, and is assumed to depend on $\phi:=\phi_{1}+\cdots+\phi_{N}$ only, i.e. $V_{i}(\Phi)=V(\phi)$ for $i=1, \ldots, N$, and may be given by the Richardson-Zaki [38] expression

$$
V(\phi)=(1-\phi)^{n-2} \quad \text { for } \phi \in \overline{\mathcal{D}}_{\phi_{\max }}, \quad V(\phi)=0 \quad \text { otherwise, } \quad n>2 .
$$

Expressing the velocities $v_{1}, \ldots, v_{N}$ and $v_{\mathrm{f}}$ in terms of the volume average velocity $q:=(1-\phi) v_{\mathrm{f}}+\phi_{1} v_{1}+$ $\cdots+v_{N} \phi_{N}$ and the slip velocities $u_{1}, \ldots, u_{N}$ we obtain the flux functions

$$
f_{i}(\Phi)=\phi_{i} v_{i}=q \phi_{i}+\phi_{i}\left(u_{i}-\left(\phi_{1} u_{1}+\cdots+\phi_{N} u_{N}\right)\right), \quad i=1, \ldots, N .
$$

while summing the continuity equations for the solids species and that of the fluid yields $\partial_{x} q=0$, i.e., $q=0$ for batch settling in a closed vessel. Then, inserting (3.1) into (3.3), assuming that (for example, after rescaling $x)$ the constant $g /\left(18 \mu_{\mathrm{f}}\right)$ equals one and recalling the ordering

$$
d_{1}=1 \geq d_{2} \geq \cdots \geq d_{N-1} \geq d_{N},
$$

we obtain

$$
f_{i}(\Phi)=\phi_{i} v_{i}(\Phi), \quad v_{i}(\Phi)=\left[\left(\varrho_{i}-\bar{\varrho}^{\mathrm{T}} \Phi\right) d_{i}^{2} V_{i}(\Phi)-\sum_{m=1}^{N} \phi_{m} d_{m}^{2}\left(\bar{\varrho}_{m}-\bar{\varrho}^{\mathrm{T}} \Phi\right) V_{m}(\Phi)\right], \quad i=1, \ldots, N .
$$

Under the assumption $V_{i}(\Phi)=V(\phi)$ for $i=1, \ldots, N$, the equation for $v_{i}$ in (3.5) assumes the form

$$
v_{i}(\Phi)=V(\phi)\left[\left(\bar{\varrho}_{i}-\bar{\varrho}^{\mathrm{T}} \Phi\right) d_{i}^{2}-\sum_{m=1}^{N} \phi_{m} d_{m}^{2}\left(\bar{\varrho}_{m}-\bar{\varrho}^{\mathrm{T}} \Phi\right)\right], \quad i=1, \ldots, N .
$$


On the other hand, for equal-density particles we have $\bar{\varrho}_{i}-\bar{\varrho}^{\mathrm{T}} \Phi=(1-\phi)\left(\varrho_{\mathrm{s}}-\varrho_{\mathrm{f}}\right)$, where $\varrho_{\mathrm{s}}$ is the uniform solids density. Hence, assuming that $t$ has been rescaled so that (3.5) becomes

$$
v_{i}(\Phi)=(1-\phi)\left[d_{i}^{2} V_{i}(\Phi)-\sum_{m=1}^{N} \phi_{m} d_{m}^{2} V_{m}(\Phi)\right], \quad i=1, \ldots, N .
$$

Clearly, combining the assumption $V_{i}(\Phi)=V(\phi)$ with that of equal particle densities leads to the flux function (1.5).

Finally, we may also consider a suspension of particles of equal size $d$ of species that differ in density only. Assuming $V_{i}(\Phi)=V(\phi)$ and that the factor $g d^{2} /\left(18 \mu_{\mathrm{f}}\right)$ is set to one, we then obtain

$$
v_{i}(\Phi)=V(\phi)\left[\bar{\varrho}_{i}+(\phi-2) \bar{\varrho}^{\mathrm{T}} \Phi\right], \quad i=1, \ldots, N .
$$

The secular equation can be employed for the hyperbolicity analysis of the MLB model in various particular instances, as we shall see in Section4.

3.2. Models based on Batchelor's approach. The MLB model is derived from the mass and linear momentum balance equations of the particle species and the fluid (see [11]). A different approach is due to Batchelor [4], which is based on the following expression for the settling velocity $v_{i}$ of spheres of species $i$, having diameter $d_{i}$, in a dilute suspension:

$$
v_{i}(\Phi)=v_{i}(0)\left(1+\mathbf{s}_{i}^{\mathrm{T}} \Phi\right), \quad i=1, \ldots, N .
$$

Here, $v_{i}(0)$ is the settling velocity of a single sphere of species $i$ in pure fluid, that is, $v_{i}(0)$ is the Stokes velocity $v_{i}(0)=-d_{i}^{2} \bar{\varrho}_{i} /\left(18 \mu_{\mathrm{f}}\right)$, and $\mathbf{s}_{i}^{\mathrm{T}}:=\left(S_{i 1}, \ldots, S_{i N}\right)$ is the $i$-th row of the matrix $\mathbf{S}=\left(S_{i j}\right)_{1 \leq i, j \leq N}$. The dimensionless sedimentation coefficients $S_{i j}$ are in general negative functions of $\lambda_{i j}:=d_{j} / d_{i}$ and $\varrho_{i j}:=\bar{\varrho}_{j} / \bar{\varrho}_{i}$, of the Péclet number $\mathcal{P}_{i j}:=\left(d_{i}+d_{j}\right)\left|v_{j}(0)-v_{i}(0)\right| /\left(4 \mathcal{D}_{i j}\right)$, and of interparticle attractive-repulsive forces. Here, $\mathcal{D}_{i j}:=(k T)\left(3 \pi \mu_{\mathrm{f}}\right)^{-1}\left(d_{i}^{-1}+d_{j}^{-1}\right)$ is the so-called relative diffusivity, where $T$ is temperature and $k$ is the Boltzmann constant $[4,6]$. The coefficients $S_{i j}$ can be calculated from the pair distribution function, which represents the statistical structure of the suspension [4]. This was done numerically by Batchelor and Wen [6] for a range of values of $\lambda=\lambda_{i j}$ and $\varrho=\varrho_{i j}$, considering the limits of either a large $\left(\mathcal{P}_{i j} \gg 1\right)$ or a small $\left(\mathcal{P}_{i j} \ll 1\right)$ Péclet number, and neglecting Brownian diffusion.

The secular equation can be employed for the hyperbolicity analysis of several models based on Batchelor's approach with equal-density particles $\left(\varrho_{i j}=1\right.$ for $\left.1 \leq i, j \leq N\right)$. In this case, after rescaling time, we may express (3.9) as

$$
v_{i}(\Phi)=d_{i}^{2}\left(1+\mathbf{s}_{i}^{\mathrm{T}} \Phi\right), \quad i=1, \ldots, N
$$

and the coefficients $S_{i j}$ can be reasonably approximated by a formula of the type

$$
S_{i j}=\sum_{l=0}^{3} \beta_{l}\left(\frac{d_{j}}{d_{i}}\right)^{l}, \quad 1 \leq i, j \leq N .
$$

We will refer to (3.10), (3.11) as the Batchelor and Wen (BW) model.

Davis and Gecol [21] were the first to approximate the numerical values of $S_{i j}$, tabulated in [6] for $\varrho_{i j}=1$ for eight different values of $\lambda_{i j}$, by an expression of the type (3.11); they obtained the coefficients

$$
\boldsymbol{\beta}^{\mathrm{T}}:=\left(\beta_{0}, \ldots, \beta_{3}\right)= \begin{cases}(-3.5,-1.1,-1.02,-0.002) & \text { for large Péclet numbers }\left(\mathcal{P}_{i j} \gg 1\right), \\ (-3.42,-1.96,-1.21,-0.013) & \text { for small Péclet numbers }\left(\mathcal{P}_{i j} \ll 1\right) .\end{cases}
$$

We observe that in both cases, $\beta_{i}<0$ for $i=0, \ldots, 3$, and that $\left|\beta_{3}\right|$ is very small. In fact, some authors utilize $\beta_{3}=0$ a priori; for example, Höfler and Schwarzer [30] fit the data from [6] for large Péclet numbers to a second-order polynomial corresponding to

$$
\boldsymbol{\beta}^{\mathrm{T}}=\left(\beta_{0}, \ldots, \beta_{3}\right)=(-3.52,-1.04,-1.03,0) .
$$

That $\left|\beta_{3}\right|$ should be a small while $\beta_{0}, \beta_{1}, \beta_{2} \leq 0$ is also supported by theoretical asymptotical result [4] stating that $S_{i j}+\varrho_{i j}\left(\lambda_{i j}^{2}+3 \lambda_{i j}+1\right) \rightarrow 0$ as $\lambda_{i j} \rightarrow \infty$, which is relevant here only for $\varrho_{i j}=1$. For a detailed discussion of the coefficients $S_{i j}$ and further data we refer to [4,6, 40,47]. Our further analysis will indeed 
rely on the negativity of the $\beta_{i}$. As we shall see shortly, setting $\beta_{3}=0$ simplifies greatly the computations involved in the hyperbolicity analysis via the secular equation.

To overcome the limitation of (3.10), and the BW approach, to dilute suspensions, other models have been proposed. Davis and Gecol [21] replace (3.10) by

$$
v_{i}(\Phi)=d_{i}^{2}\left(1+\mathbf{s}_{i}^{\mathrm{T}} \Phi-S_{i i} \phi\right)(1-\phi)^{-S_{i i}},
$$

and claimed that (3.14) could be used for size ratios $d_{N} \geq 1 / 8$. However, in [14] it is shown that for $N=2$ and $d_{2} \approx 1 / 6$, the system (1.1) based on using (3.14) exhibits unphysical instability regions for equal-density spheres. Another velocity equation that formally extends (3.10) to the whole range of concentrations was suggested by Höfler and Schwarzer [13, 29, 30]:

$$
v_{i}(\Phi)=d_{i}^{2} \exp \left(\mathbf{s}_{i}^{\mathrm{T}} \Phi+n \phi\right)(1-\phi)^{n}, \quad n \geq 0 .
$$

For $\Phi \rightarrow 0,(3.14)$ and (3.15) have the same partial derivatives as (3.10), while for $\phi \rightarrow 1$, the velocities $v_{i}$ given by (3.14) and (3.15) vanish. Moreover, for the HS model it is straightforward to verify (see [14]) that $I_{2}>0$ for arbitrary non-positive Batchelor matrices $\mathbf{S}$ and $N=2$. Furthermore, based on numerical tests, it was conjectured in [14] that the model based on (3.15) would be stable also for $N=3$. The present work confirms this conjecture and shows that the model is stable for arbitrary $N$, provided that for a given vector of coefficients $\boldsymbol{\beta}$, the quantities $d_{N}$ and $\Phi$ satisfy some mild conditions.

\section{Hyperbolicity anAlysis VIA the SECUlar EQUATION}

Since our analysis should be general with respect to the number of species $N$ and the particle size classes $d_{1}, \ldots, d_{N}$, we employ the smallest normalized particle size $d_{N}$ as the only scalar parameter that characterizes the width of the particle size distribution. We always assume the ordering of particle sizes (3.4). This means that for equal-density particles, $i>j$ for $i, j \in\{1, \ldots, N\}$ is equivalent to $d_{i}<d_{j}$.

4.1. The Masliyah-Lockett-Bassoon (MLB) model. The MLB model for equal-density spheres (1.1), (1.5) is known to be strictly hyperbolic for all $N$ and all $\Phi \in \mathcal{D}_{\phi_{\max }}$. A proof of this fact was obtained in [11], by deriving an explicit formula for the characteristic polynomial of the Jacobian matrix $\mathcal{J}_{\mathbf{f}}(\Phi)$. In [23], a considerably shorter proof of this same fact was provided by using the secular equation.

To illustrate the use of the secular equation to obtain strict hyperbolicity, we consider the MLB model for species of identical density in a more general case, considered in [3]. Basson et al. [3] recently analyzed the variant of the MLB model for equal-density spheres based on (3.7) with the following hindered settling factors:

$$
V_{i}(\phi)=(1-\phi)^{n_{i}-2} \quad \text { for } \phi \in \overline{\mathcal{D}}_{\phi_{\max }}, \quad V_{i}(\phi)=0 \quad \text { otherwise, } \quad n_{i}>2, \quad n_{i} \leq n_{j} \text { for } i<j .
$$

In [3], an analysis of the characteristic polynomial of $\mathcal{J}_{\mathbf{f}}(\Phi)$ similar to that of [11] led to the conclusion that the model is still strictly hyperbolic for all $N$ and $\Phi \in \mathcal{D}_{\phi_{\max }}$. Here we provide a much shorter proof of this fact using the secular equation.

Lemma 4.1. The MLB model for equal-density spheres (1.1), (3.7) and the hindered settling factors (4.1) is strictly hyperbolic for all $\Phi \in \mathcal{D}_{\phi_{\max }}$. Its eigenvalues $\lambda_{i}=\lambda_{i}(\Phi)$ satisfy the interlacing property

$$
\begin{gathered}
M_{1}(\Phi)<\lambda_{N}(\Phi)<v_{N}(\Phi)<\lambda_{N-1}(\Phi)<v_{N-1}(\Phi)<\cdots<\lambda_{1}(\Phi)<v_{1}(\Phi), \\
M_{1}(\Phi):=d_{N}^{2} V_{N}(\Phi)+\sum_{j=1}^{N} d_{j}^{2} \phi_{j}\left((1-\phi) V_{j}^{\prime}(\phi)-2 V_{j}(\phi)\right) .
\end{gathered}
$$

The right and left eigenvectors of $\mathcal{J}_{\mathbf{f}}(\Phi)$, respectively denoted by $\mathbf{x}$ and $\mathbf{y}$, that correspond to a root $\lambda$ of the secular equation are

$$
\mathbf{x}_{i}=\frac{1}{v_{i}-\lambda}\left[b_{i, 1} \sum_{k=1}^{N} \frac{a_{k, 1} b_{k, 2}}{v_{k}-\lambda}-b_{i, 2}\left(1+\sum_{k=1}^{N} \frac{a_{k, 1} b_{k, 1}}{v_{k}-\lambda}\right)\right], \quad i=1, \ldots, N,
$$




$$
\mathbf{y}_{i}=\frac{1}{v_{i}-\lambda}\left[a_{i, 1} \sum_{k=1}^{N} \frac{b_{k, 1} a_{k, 2}}{v_{k}-\lambda}-a_{i, 2}\left(1+\sum_{k=1}^{N} \frac{b_{k, 1} a_{k, 1}}{v_{k}-\lambda}\right)\right], \quad i=1, \ldots, N
$$

where

$$
b_{i, 1}=\phi_{i} d_{i}^{2} V_{i}^{\prime}(\phi), \quad b_{i, 2}=-\phi_{i}, \quad a_{i, 1}=1, \quad a_{i, 2}=\sum_{j=1}^{N} d_{j}^{2} V_{j}^{\prime}(\phi) \phi_{j}+d_{i}^{2} V_{i}(\phi) .
$$

Proof. In this case, we have $m=2$, and the velocities can be expressed by $v_{i}=V_{i}\left(p_{1}\right) d_{i}^{2}-p_{2}$, where $p_{1}:=\phi=\phi_{1}+\cdots+\phi_{N}$ and $p_{2}=d_{1}^{2} V_{1}(\phi) \phi_{1}+\cdots+d_{N}^{2} V_{N}(\phi) \phi_{N}$. To compute the expressions in (2.14), we need

$$
\begin{gathered}
v_{l}-v_{i}=V_{l}(\phi) d_{l}^{2}-V_{i}(\phi) d_{i}^{2}, \quad \frac{\partial v_{i}}{\partial p_{1}}=d_{i}^{2} V_{i}^{\prime}(\phi), \quad \frac{\partial v_{i}}{\partial p_{2}}=-1, \quad \frac{\partial p_{1}}{\partial \phi_{i}}=1, \quad \frac{\partial p_{2}}{\partial \phi_{i}}=\sum_{j=1}^{N} d_{j}^{2} V_{j}^{\prime}(\phi) \phi_{j}+d_{i}^{2} V_{i}(\phi) ; \\
\operatorname{det}\left(\frac{\partial v_{i, l}}{\partial p_{1,2}}\right)=d_{l}^{2} V_{l}^{\prime}(\phi)-d_{i}^{2} V_{i}^{\prime}(\phi), \quad \operatorname{det}\left(\frac{\partial p_{1,2}}{\partial \phi_{i, l}}\right)=d_{l}^{2} V_{l}(\phi)-d_{i}^{2} V_{i}(\phi) .
\end{gathered}
$$

In this way, we obtain

$$
\begin{aligned}
\gamma_{1, i} & =\frac{\partial v_{i}}{\partial p_{1}} \frac{\partial p_{1}}{\partial \phi_{j}}+\frac{\partial v_{i}}{\partial p_{2}} \frac{\partial p_{2}}{\partial \phi_{j}}=d_{i}^{2}\left(V_{i}^{\prime}(\phi)-V_{i}(\phi)\right)-\sum_{j=1}^{N} d_{j}^{2} V_{j}^{\prime}(\phi) \phi_{j}, \\
\gamma_{2, i} & =\sum_{\substack{j=1 \\
j \neq i}}^{N} \frac{\phi_{j}}{v_{j}-v_{i}} \operatorname{det}\left(\frac{\partial v_{i, j}}{\partial p_{1,2}}\right) \operatorname{det}\left(\frac{\partial p_{1,2}}{\partial \phi_{i, j}}\right)=\sum_{\substack{j=1 \\
j \neq i}}^{N} \phi_{j}\left(d_{j}^{2} V_{j}^{\prime}(\phi)-d_{i}^{2} V_{i}^{\prime}(\phi)\right),
\end{aligned}
$$

which finally leads to $\gamma_{i}=\left(V_{i}^{\prime}(\phi)(1-\phi)-V_{i}(\phi)\right) \phi_{i} d_{i}^{2}$. Due to (1.6), we obtain $\gamma_{i}<0$ for all $i=1, \ldots, N$ and $\Phi \in \mathcal{D}_{\phi_{\max }}$. The interlacing property and the form of $M_{1}$ follow from Corollary 2.1. We deduce (4.2) by considering $\lambda=\lambda_{j}$, taking $\boldsymbol{\xi}=\left(-\left[\mathbf{a}_{1}, \mathbf{b}_{2}\right], 1+\left[\mathbf{a}_{1}, \mathbf{b}_{1}\right]\right) \in \mathbb{R}^{2}$ as solution of the first of the two (equivalent) equations in (2.12) and substituting into (2.13). Since the left eigenvectors of $\mathcal{J}_{\mathbf{f}}=\mathbf{D}+\mathbf{B} \mathbf{A}^{\mathrm{T}}$ are the right eigenvectors of $\mathcal{J}_{\mathbf{f}}^{\mathrm{T}}=\mathbf{D}+\mathbf{A} \mathbf{B}^{\mathrm{T}}$, (4.3) can be deduced from (4.2) by interchanging the roles of $\mathbf{A}$ and $\mathbf{B}$.

For equal-sized particles, which differ in density only, $\mathcal{D}_{\phi_{\max }}$ has in general a sub-region with lack of hyperbolicity $[12,14]$. In this case, the quantities $\gamma_{i}$ will in general not have a definite sign, but we may still employ the secular equation to estimate the size of the hyperbolicity region of $\mathcal{D}_{\phi_{\max }}$.

Lemma 4.2. The MLB model for equal-sized heavy spheres (1.1), (3.8), where $\bar{\varrho}_{1}>\bar{\varrho}_{2}>\cdots>\bar{\varrho}_{N}>0$ and the hindered settling factor $V(\phi)$ is given by (3.2), is strictly hyperbolic for all $\Phi \in \mathcal{D}_{\phi_{*}} \subset \mathcal{D}_{1}$, where

$$
\phi_{*}=\frac{n \bar{\varrho}_{N}}{\bar{\varrho}_{1}+\bar{\varrho}_{N}(n-1)}=\frac{n}{n+\gamma-1}, \quad \gamma=\bar{\varrho}_{1} / \bar{\varrho}_{N}>1 .
$$

Proof. In this case, we have $v_{i}=V\left(p_{1}\right)\left(\bar{\varrho}_{i}+\left(p_{1}-2\right) p_{2}\right)$, where $p_{1}=\phi$ and $p_{2}=\bar{\varrho}^{\mathrm{T}} \Phi$. This implies

$$
\begin{gathered}
v_{j}-v_{i}=V(\phi)\left(\bar{\varrho}_{j}-\bar{\varrho}_{i}\right), \quad \frac{\partial v_{i}}{\partial p_{1}}=V^{\prime}(\phi)\left(\bar{\varrho}_{i}+(\phi-2) p_{2}\right)+V(\phi) p_{2}, \quad \frac{\partial v_{i}}{\partial p_{2}}=V(\phi)(\phi-2), \\
\frac{\partial p_{1}}{\partial \phi_{i}}=1, \quad \frac{\partial p_{2}}{\partial \phi_{i}}=\bar{\varrho}_{i} ; \quad \operatorname{det}\left(\frac{\partial v_{i j}}{\partial p_{12}}\right)=V(\phi) V^{\prime}(\phi)(\phi-2)\left(\bar{\varrho}_{i}-\bar{\varrho}_{j}\right), \quad \operatorname{det}\left(\frac{\partial p_{12}}{\partial \phi_{i j}}\right)=\bar{\varrho}_{j}-\bar{\varrho}_{i} .
\end{gathered}
$$

In this way, we get

$$
\gamma_{1, i}=V^{\prime}(\phi)\left(\bar{\varrho}_{i}+(\phi-2) p_{2}\right)+V(\phi)\left(p_{2}+(\phi-2) \varrho_{i}\right), \quad \gamma_{2, i}=V^{\prime}(\phi)(\phi-2)\left(\phi \bar{\varrho}_{i}-p_{2}\right),
$$

which for $V(\phi)$ given by (3.2) yields

$$
\gamma_{i}=-\phi_{i}(1-\phi)^{n-2}\left[\bar{\varrho}_{i}(1+(n-1)(1-\phi))-p_{2}\right] .
$$


Taking into account the ordering $\bar{\varrho}_{1}>\cdots>\bar{\varrho}_{N}$, we have $\gamma_{i}<0$ if $\phi<1$ and $p_{2}<\bar{\varrho}_{i}\left(1+(n-1)\left(1-p_{1}\right)\right)$, for all $i=1, \ldots, N$, or equivalently, $0<\psi(\Phi):=-\bar{\varrho}^{\mathrm{T}} \Phi+\bar{\varrho}_{N}(1+(n-1)(1-\phi))$ for all $\Phi \in \overline{\mathcal{D}}_{\phi_{*}}$. In order to find conditions on $\phi_{*}$ for ensuring that $\psi(\phi)>0, \forall \phi \in\left[0, \phi_{\max }\right]$, we may consider the linear optimization programme

$$
\min _{\phi_{j} \geq 0, \phi \leq \phi_{*}} \psi(\Phi)
$$

It is straightforward to see that the solution of (4.6) is attained at a vertex of $\overline{\mathcal{D}}_{\phi_{*}}$. We then obtain

$$
\begin{aligned}
\min _{\Phi \in \overline{\mathcal{D}}_{\phi_{*}}} \psi(\Phi) & =\min \left\{\psi\left((0, \ldots, 0)^{\mathrm{T}}\right), \min \left\{\psi\left(\phi_{*} \mathbf{e}_{1}\right), \ldots, \psi\left(\phi_{*} \mathbf{e}_{N}\right)\right\}\right\} \\
& =\min _{1 \leq j \leq N}\left\{-\bar{\varrho}_{j} \phi_{*}+\bar{\varrho}_{N}\left(1+(n-1)\left(1-\phi_{*}\right)\right)\right\}=-\bar{\varrho}_{1} \phi_{*}+\bar{\varrho}_{N}\left(1+(n-1)\left(1-\phi_{*}\right)\right) .
\end{aligned}
$$

This directly gives the bound (4.5) that ensures strict hyperbolicity in $\mathcal{D}_{\phi_{\max }}$.

4.2. Preliminaries for the BW and HS models. These two models can be expressed as

$$
v_{i}(\Phi)=d_{i}^{2} \varphi\left(\mathbf{s}_{i}^{\mathrm{T}} \Phi+n \phi\right)(1-\phi)^{n}, \quad i=1, \ldots, N,
$$

where $\varphi(z)=1+z, n=0$ for the BW model, and $\varphi(z)=\exp (z), n \geq 0$, arbitrary, for the HS model. We define

$$
\mathbf{a}_{\nu}:=\mathbf{d}_{\nu-1}^{\mathrm{T}}:=\left(d_{1}^{\nu-1}, d_{2}^{\nu-1}, \ldots, d_{N}^{\nu-1}\right), \quad p_{\nu}:=\mathbf{a}_{\nu}^{\mathrm{T}} \Phi, \quad \nu=1, \ldots, 4,
$$

and write

$$
\mathbf{s}_{i}^{\mathrm{T}} \Phi=\sum_{j=1}^{N}\left(\sum_{\nu=0}^{3} \beta_{\nu}\left(\frac{d_{j}}{d_{i}}\right)^{\nu}\right) \phi_{j}=\sum_{\nu=0}^{3} \frac{\beta_{\nu}}{d_{i}^{\nu}} \mathbf{a}_{\nu}^{\mathrm{T}} \Phi=\sum_{\nu=0}^{3} \frac{\beta_{\nu}}{d_{i}^{\nu}} p_{\nu+1}, \quad i=1, \ldots, N .
$$

In this paper we shall analyze only the case $\beta_{3}=0$, for which $m=3$. We may then express (4.7) as

$$
v_{i}(\Phi)=v_{i}\left(p_{1}, \ldots, p_{3}\right)=d_{i}^{2} \varphi\left(\left(\beta_{0}+n\right) p_{1}+\beta_{1} d_{i}^{-1} p_{2}+\beta_{2} d_{i}^{-2} p_{3}\right)\left(1-p_{1}\right)^{n}, \quad i=1, \ldots, N .
$$

Let us write $p_{1}=\phi$, and define $\eta_{i}:=\varphi\left(\mathbf{s}_{i}^{\mathrm{T}} \Phi+n \phi\right)$ and $\eta_{i}^{\prime}:=\varphi^{\prime}\left(\mathbf{s}_{i}^{\mathrm{T}} \Phi+n \phi\right)$ for $i=1, \ldots, N$, where $\varphi^{\prime}(z):=d \varphi(z) / d z$. Taking into account that for the BW and HS models either $n=0$ or $\eta_{i}^{\prime}=\eta_{i}$, we readily compute here that the quantities $\alpha_{i}^{k}=\partial p_{k} / \partial \phi_{i}$ and $\beta_{i}^{k}=\phi_{i} \partial v_{i} / \partial p_{k}$ are given by

$$
\alpha_{i}^{k}=d_{i}^{k-1}, \quad \beta_{i}^{k}=d_{i}^{3-k} \phi_{i}(1-\phi)^{n} \tilde{\beta}_{k-1} \eta_{i}^{\prime}, \quad \tilde{\beta}_{0}=\beta_{0}-\frac{n \phi}{1-\phi}, \quad \tilde{\beta}_{k}=\beta_{k}, \quad k=1,2 .
$$

We now calculate the products $\alpha_{I}^{J} \beta_{I}^{J}$ of the determinants $\alpha_{I}^{J}:=\operatorname{det} \mathbf{A}^{I, J}$ and $\beta_{I}^{J}:=\operatorname{det} \mathbf{B}^{I, J}$ in the formula (2.14) for $m=3$,

$$
\gamma_{i}=\alpha_{i}^{1} \beta_{i}^{1}+\alpha_{i}^{2} \beta_{i}^{2}+\alpha_{i}^{3} \beta_{i}^{3}+\sum_{\substack{j=1 \\ j \neq i}}^{N} \frac{\alpha_{i j}^{12} \beta_{i j}^{12}+\alpha_{i j}^{13} \beta_{i j}^{13}+\alpha_{i j}^{23} \beta_{i j}^{23}}{v_{j}-v_{i}}+\sum_{\substack{j, k=1 \\ i \neq j<k \neq i}}^{N} \frac{\alpha_{i j k}^{123} \beta_{i j k}^{123}}{\left(v_{k}-v_{i}\right)\left(v_{j}-v_{i}\right)},
$$

which is written out here in some detail for the ease of keeping track of the terms to be evaluated. Moreover, we adopt the convention that sums over a void index range are zero, and utilize the following notation:

$$
\pi_{i j k}:=\left(d_{j}-d_{i}\right)\left(d_{k}-d_{i}\right)\left(d_{k}-d_{j}\right) .
$$

We then obtain

$$
\begin{aligned}
\alpha_{i}^{k} \beta_{i}^{k} & =d_{i}^{2} \phi_{i}(1-\phi)^{n} \tilde{\beta}_{k-1} \eta_{i}^{\prime}, \quad k=1,2,3 \\
\alpha_{i j}^{p, p+q} \beta_{i j}^{p, p+q} & =-\left(d_{i} d_{j}\right)^{2-q} \phi_{i} \phi_{j}(1-\phi)^{2 n} \eta_{i}^{\prime} \eta_{j}^{\prime} \tilde{\beta}_{p-1} \tilde{\beta}_{p+q-1}\left(d_{i}^{q}-d_{j}^{q}\right)^{2}, \quad q=1,2, \\
\alpha_{i j k}^{123} \beta_{i j k}^{123} & =-\phi_{i} \phi_{j} \phi_{k}(1-\phi)^{3 n} \eta_{i}^{\prime} \eta_{j}^{\prime} \eta_{k}^{\prime} \tilde{\beta}_{0} \beta_{1} \beta_{2} \pi_{i j k}^{2} .
\end{aligned}
$$


4.3. The Batchelor and Wen (BW) model. We first show that for a sufficiently dilute suspension (i.e., $\Phi$ is close to zero in a sense made precise below), the BW model is stable by demonstrating that $\gamma_{i}$ has a definite sign, at least under certain mild restrictions on the parameters $d_{N}$ and $\boldsymbol{\beta}$, and if the components of $\Phi$ are sufficiently small. To this end, we rewrite (4.10) as $\gamma_{i}=\phi_{i}\left(\mathcal{S}_{1, i}+\mathcal{S}_{2, i}+\mathcal{S}_{3, i}\right)$. Inserting the expressions (4.11) and (4.12), with $n=0, \tilde{\beta}_{k}=\beta_{k}, \eta_{i}^{\prime}=1$, into (4.10) and defining $\hat{\eta}_{i}:=1+\mathbf{s}_{i}^{\mathrm{T}} \Phi$, we obtain

$$
\begin{aligned}
& \mathcal{S}_{1, i}:=d_{i}^{2}\left(\beta_{0}+\beta_{1}+\beta_{2}\right), \\
& \mathcal{S}_{2, i}:=\sum_{\substack{j=1 \\
j \neq i}}^{N} \frac{\phi_{j}}{d_{j}^{2} \hat{\eta}_{j}-d_{i}^{2} \hat{\eta}_{i}}\left(-\beta_{0} \beta_{1} d_{i} d_{j}\left(d_{j}-d_{i}\right)^{2}-\beta_{0} \beta_{2}\left(d_{j}^{2}-d_{i}^{2}\right)^{2}-\beta_{1} \beta_{2} d_{i} d_{j}\left(d_{j}-d_{i}\right)^{2}\right), \\
& \mathcal{S}_{3, i}:=-\beta_{0} \beta_{1} \beta_{2} \sum_{\substack{j, k=1 \\
i \neq j<k \neq i}}^{N} \frac{\phi_{j} \phi_{k} \pi_{i j k}^{2}}{\left(d_{k}^{2} \hat{\eta}_{k}-d_{i}^{2} \hat{\eta}_{i}\right)\left(d_{j}^{2} \hat{\eta}_{j}-d_{i}^{2} \hat{\eta}_{i}\right)} .
\end{aligned}
$$

Since $\beta_{i} \leq 0$ for $i=0,1,2$ and at least one $\beta_{i}$ is negative, we see that $\mathcal{S}_{1, i}<0$ for $\phi_{i}>0$; moreover, here $\mathcal{S}_{1, i}$ is independent of $\Phi$ or $N$. Consequently, we now show that $\gamma_{i}<0$ for all $i=1, \ldots, N$ by possibly imposing further conditions on the parameters $d_{N}, \boldsymbol{\beta}$ and $\Phi$. Our strategy is based on splitting the sums of (4.13) into positive and negative parts (produced by summands of the corresponding sign), estimating the contributions of positive sign, and then showing that these estimates ensure that $\gamma_{i}<0$. To this end, suppose that there is a constant $\theta \geq 1$ such that

$$
-\mathbf{s}_{i}^{\mathrm{T}} \Phi \leq \frac{1}{1+\theta} \quad \text { for all } i=1, \ldots, N
$$

Clearly, this condition is satisfied if and only if the inequality for $i=N$ is satisfied, i.e.,

$$
-\mathbf{s}_{N}^{\mathrm{T}} \Phi=\sum_{j=1}^{N}\left(-\sum_{\nu=0}^{2} \frac{\beta_{\nu} d_{j}^{\nu}}{d_{N}^{\nu}}\right) \phi_{j} \leq \frac{1}{1+\theta} .
$$

(This is a combined condition on the choices of $d_{N}, \boldsymbol{\beta}$ and $\Phi$, which we will discuss after stating the main result for this model.) This implies that $1+\mathbf{s}_{i}^{\mathrm{T}} \Phi \geq-\theta \mathbf{s}_{i}^{\mathrm{T}} \Phi$ for all $i=1, \ldots, N$, i.e., it is a positive lower bound for the velocities $v_{i}$, and we then know that for $i<j$, the following inequalities are valid:

$$
0<\left(d_{i}^{2} \hat{\eta}_{i}-d_{j}^{2} \hat{\eta}_{j}\right)^{-1} \leq\left(\left(1+\mathbf{s}_{j}^{\mathrm{T}} \Phi\right)\left(d_{i}^{2}-d_{j}^{2}\right)\right)^{-1} \leq\left(-\theta\left(d_{i}^{2}-d_{j}^{2}\right) \sum_{\nu=0}^{2} \frac{\beta_{\nu}}{d_{j}^{\nu}} \mathbf{d}_{\nu}^{\mathrm{T}} \Phi\right)^{-1} .
$$

Clearly, we may further estimate the last term in (4.16) by omitting some of the summands.

Lemma 4.3. If (4.15) is satisfied then we have the following inequalities:

$$
\begin{aligned}
& \mathcal{S}_{2, i} \leq-d_{i}^{2} \theta^{-1}\left(2 \beta_{0}+\beta_{2}\right), \\
& \mathcal{S}_{3, i} \leq-d_{i}^{2} \theta^{-2}\left(2 \beta_{0}\right) .
\end{aligned}
$$

Proof. Since $\hat{\eta}_{i}>\hat{\eta}_{j}$ for $i<j$, the summands of $\mathcal{S}_{2, i}$ with $j<i$ and $j>i$ are negative and positive, respectively; let us denote the corresponding partial sums by $\mathcal{S}_{2, i}^{-} \leq 0$ and $\mathcal{S}_{2, i}^{+} \geq 0$, with $\mathcal{S}_{2, i}=\mathcal{S}_{2, i}^{-}+\mathcal{S}_{2, i}^{+}$. Our aim is to bound $\mathcal{S}_{2, i}^{+}$in such a way that this quantity is compensated by the (negative) terms of $\mathcal{S}_{1}$.

Let us now turn to $\mathcal{S}_{2, i}^{+}$. We here get

$$
\mathcal{S}_{2, i}^{+} \leq-\frac{1}{\theta} \sum_{j=i+1}^{N}\left\{\frac{\beta_{0} \beta_{1} d_{i}\left(d_{i}-d_{j}\right)^{2} d_{j}^{2} \phi_{j}}{\left(d_{i}^{2}-d_{j}^{2}\right) \beta_{1} \mathbf{d}_{1}^{\mathrm{T}} \Phi}+\frac{\beta_{0} \beta_{2}\left(d_{i}+d_{j}\right)^{2}\left(d_{i}-d_{j}\right)^{2} d_{j}^{2} \phi_{j}}{\left(d_{i}^{2}-d_{j}^{2}\right) \beta_{2} \mathbf{d}_{2}^{\mathrm{T}} \Phi}+\frac{\beta_{1} \beta_{2} d_{i}\left(d_{i}-d_{j}\right)^{2} d_{j}^{2} \phi_{j}}{\beta_{1}\left(d_{i}^{2}-d_{j}^{2}\right) \mathbf{d}_{1}^{\mathrm{T}} \Phi}\right\} .
$$

Consequently, since $d_{i}>d_{j}$ for $j>i$, we obtain from (4.19) the following inequality:

$$
\mathcal{S}_{2, i}^{+} \leq-\frac{d_{i}^{2}}{\theta} \sum_{j=i+1}^{N}\left\{\beta_{0}\left(\frac{d_{j} \phi_{j}}{\mathbf{d}_{1}^{\mathrm{T}} \Phi}+\frac{d_{j}^{2} \phi_{j}}{\mathbf{d}_{2}^{\mathrm{T}} \Phi}\right)+\beta_{2} \frac{d_{j} \phi_{j}}{\mathbf{d}_{1}^{\mathrm{T}} \Phi}\right\},
$$


which implies (4.17), given that

$$
\frac{d_{i+1}^{p} \phi_{i+1}+\cdots+d_{N}^{p} \phi_{N}}{d_{1}^{p} \phi_{1}+\cdots+d_{N}^{p} \phi_{N}} \leq 1, \quad p=1,2 .
$$

Since only those summands of $\mathcal{S}_{3, i}$ are positive for which either $i<j$ and $i<k$ or $i>j$ and $i>k$, we rewrite $\mathcal{S}_{3, i}$ as $\mathcal{S}_{3, i}=\mathcal{S}_{3, i}^{-}+\mathcal{S}_{3, i}^{+, 1}+\mathcal{S}_{3, i}^{+, 2}$, where $\mathcal{S}_{3, i}^{-}<0, \mathcal{S}_{3, i}^{+, 1}>0$ and $\mathcal{S}_{3, i}^{+, 2}>0$, and $\mathcal{S}_{3, i}^{+, 1}$ and $\mathcal{S}_{3, i}^{+, 2}$ are the partial of $\mathcal{S}_{3, i}$ for which $j>i, k>i$ and $k \neq j$ and $j<i, k<i$ and $k \neq j$, respectively.

Applying several versions of (4.16) to both factors in the denominator of the summands of $\mathcal{S}_{3, i}^{+, 1}$, we obtain

$$
\mathcal{S}_{3, i}^{+, 1} \leq-\frac{1}{\theta^{2}} \sum_{\substack{j, k=i+1 \\ j<k}}^{N} \frac{\beta_{0} \pi_{i j k}^{2} d_{j} \phi_{j} d_{k}^{2} \phi_{k}}{\left(d_{k}^{2}-d_{i}^{2}\right)\left(d_{j}^{2}-d_{i}^{2}\right) \mathbf{d}_{1}^{\mathrm{T}} \Phi \mathbf{d}_{2}^{\mathrm{T}} \Phi} .
$$

Noting that for $j, k>i$, we have that

$$
\frac{\pi_{i j k}^{2}}{\left(d_{i}^{2}-d_{j}^{2}\right)\left(d_{i}^{2}-d_{k}^{2}\right)} \leq d_{i}^{2}, \quad \sum_{\substack{j, k=i+1 \\ j<k}}^{N} d_{j} \phi_{j} d_{k}^{2} \phi_{k} \leq \mathbf{d}_{1}^{\mathrm{T}} \Phi \mathbf{d}_{2}^{\mathrm{T}} \Phi,
$$

we finally obtain the inequality

$$
\mathcal{S}_{3, i}^{+, 1} \leq-\beta_{0} \frac{d_{i}^{2}}{\theta^{2}}
$$

Furthermore, the version of (4.16) with the roles of $i$ and $j$ interchanged shows that

$$
\begin{aligned}
\mathcal{S}_{3, i}^{+, 2} & \leq-\frac{1}{\theta^{2}} \sum_{\substack{j, k=1 \\
k<j}}^{i-1} \frac{\beta_{0} \pi_{i j k}^{2} d_{i} \phi_{j} d_{i}^{2} \phi_{k}}{\left(d_{k}^{2}-d_{i}^{2}\right)\left(d_{j}^{2}-d_{i}^{2}\right) \mathbf{d}_{1}^{\mathrm{T}} \Phi \mathbf{d}_{2}^{\mathrm{T}} \Phi} \leq-\frac{\beta_{0}}{\theta^{2} \mathbf{d}_{1}^{\mathrm{T}} \Phi \mathbf{d}_{2}^{\mathrm{T}} \Phi} \sum_{\substack{j, k=1 \\
k<j}}^{i-1}\left(d_{k}-d_{j}\right)^{2} d_{i} \phi_{j} d_{i}^{2} \phi_{k} \\
& \leq-\frac{d_{i}^{2} \beta_{0}}{\theta^{2} \mathbf{d}_{1}^{\mathrm{T}} \Phi \mathbf{d}_{2}^{\mathrm{T}} \Phi} \sum_{\substack{j, k=1 \\
k<j}}^{i-1} d_{k}^{2} \phi_{k} d_{j} \phi_{j} \leq-\beta_{0} \frac{d_{i}^{2}}{\theta^{2}} .
\end{aligned}
$$

Combining this with (4.21) we obtain (4.18).

Corollary 4.1. For the BW model, the following inequality is valid:

$$
\mathcal{S}_{1, i}+\mathcal{S}_{2, i}+\mathcal{S}_{3, i} \leq d_{i}^{2} M(\theta, \boldsymbol{\beta})
$$

where we define the function

$$
M(\theta, \boldsymbol{\beta}):=\left(1-2 \theta^{-1}-2 \theta^{-2}\right) \beta_{0}+\beta_{1}+\left(1-\theta^{-1}\right) \beta_{2} .
$$

Proof. Combining the inequalities (4.17) and (4.18) we obtain (4.22) and (4.23). Each of the inequalities (4.17) and (4.18) estimates a non-negative sum from above, and therefore remains valid if the respective sum runs over a void index range, and is therefore zero. Consequently, (4.22) and (4.23) hold for arbitrary numbers of species $N$.

We have proven the following theorem.

Theorem 4.1. Assume that $\theta$ is chosen such that the inequality

$$
M(\theta, \boldsymbol{\beta})<0
$$

is satisfied, where $M(\theta, \boldsymbol{\beta})$ is defined in (4.23). If the maximum solids concentration $\phi_{\max }$ is chosen such that the inequality (4.15) is satisfied for all $\Phi \in \mathcal{D}_{\phi_{\max }}$ for this value of $\theta$, then $\gamma_{i}<0$ for $i=1, \ldots, 4$ and $\Phi \in \mathcal{D}_{\phi_{\max }}$, i.e., the model equations are strictly hyperbolic on $\mathcal{D}_{\phi_{\max }}$. 
(a)

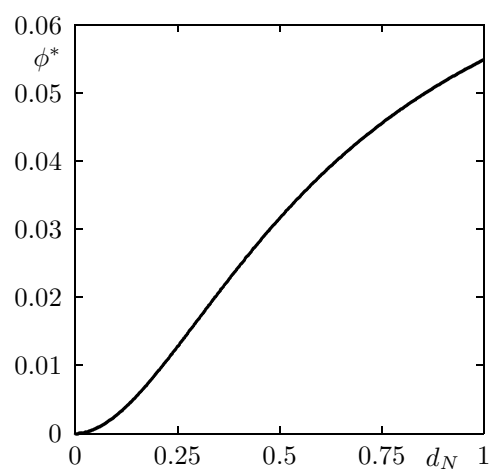

(b)

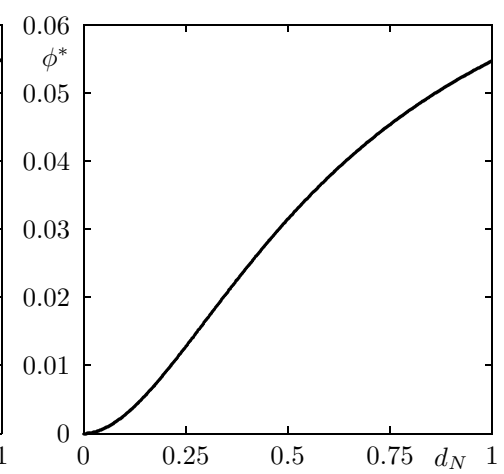

(c)

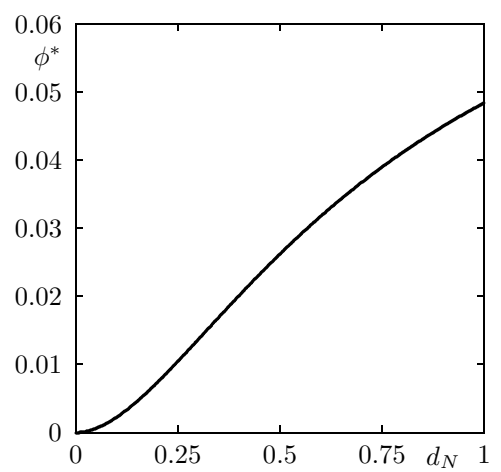

FIG. 1. Maximum total concentrations $\phi^{*}$ for which hyperbolicity of the BW model is ensured (a) for the coefficients (3.13) (with $\beta_{3}=0$ ), (b) for $\beta_{0}=-3.5, \beta_{1}=-1.1, \beta_{2}=$ -1.02 (according to (3.12) for large Péclet numbers) and $\beta_{3}=0$, and (c) for $\beta_{0}=-3.42$, $\beta_{1}=-1.96, \beta_{2}=-1.21$ (according to (3.12) for small Péclet numbers) and $\beta_{3}=0$.

The advantage of introducing the parameter $\theta$ becomes apparent now, and is related to the fact that the BW model is valid for dilute suspensions only. Suppose that we choose an admissible value of $\theta$, then (4.15) can hold either for a dilute suspension, i.e. $\phi$ is small, but for a large range of coefficients $\boldsymbol{\beta}$, or we consider relatively small (in absolute value) coefficients $\boldsymbol{\beta}$ and obtain a hyperbolicity (stability) result valid up to relatively large concentrations. Our analysis also shows that for $N=2$, we additionally have $\mathcal{S}_{3, i}=0$ and the terms in which we divide by $\theta^{2}$ are zero.

To illustrate the predictions of Theorem 4.1, let us first consider the coefficients $\boldsymbol{\beta}$ (with $\beta_{3}=0$ ) given by $(3.13)$. Since $M(\theta, \boldsymbol{\beta})$ is a strictly decreasing function of $\theta$, it is sufficient to solve $M(\theta, \boldsymbol{\beta})=0$ for $\theta$ to conclude that in this case, $M(\theta, \boldsymbol{\beta})<0$ for $\theta>\theta_{\min } \approx 2.259847$, i.e., the set of admissible values of $\theta$ is $\left(\theta_{\min }, \infty\right)$. In this case the hyperbolicity of the BW model is ensured for those vectors $\Phi$ that satisfy (4.15) with a strict inequality and $\theta=\theta_{\min }$. The sharp evaluation of this inequality requires specifying $d_{2}, \ldots, d_{N}$. However, if we only wish to determine the largest value $\phi^{*}$ of the total concentration $\phi$ up to which we can guarantee hyperbolicity, then we can rewrite the left-hand side of (4.15) as $\sigma_{1} \phi_{1}+\cdots+\sigma_{N} \phi_{N}$, where we define $\sigma_{j}:=-\beta_{0}-\beta_{1} d_{j} d_{N}^{-1}-\beta_{2} d_{j}^{2} d_{N}^{-2}$. Then the sought concentration $\phi^{*}$ solves the problem "minimize $\phi$ subject to $\sigma_{1} \phi_{1}+\cdots+\sigma_{N} \phi_{N}=\left(1+\theta_{\text {min }}\right)^{-1}$ ". Expressing $\phi_{1}$ in terms of $\phi_{2}, \ldots, \phi_{N}$ and $\phi$, we can rewrite this equation as

$$
\phi=\left(1-\sigma_{1}^{-1} \sigma_{2}\right) \phi_{2}+\cdots+\left(1-\sigma_{1}^{-1} \sigma_{N}\right) \phi_{N}+\sigma_{1}^{-1}\left(1+\theta_{\min }\right)^{-1} .
$$

Since $\sigma_{1}>\sigma_{2}>\cdots>\sigma_{N}$, the coefficients of $\phi_{2}, \ldots, \phi_{N}$ on the right-hand side are all positive, and the minimum $\phi^{*}$ of $\phi$ is attained for $\phi_{2}=\cdots=\phi_{N}=0$. Its value is $\phi^{*}=\sigma_{1}^{-1}\left(1+\theta_{\min }\right)^{-1}$. Figure 1 (a) shows a plot of $\phi^{*}$ as a function of $d_{N}$ for this case.

Finally, for the purpose of illustration, let us consider the coefficients $\boldsymbol{\beta}$ given (3.12) for large or small Péclet numbers, but where we replace the respective values of $\beta_{3}$ by zero. In these cases, we obtain the respective values $\theta_{\min } \approx 2.252800$ and $\theta_{\min } \approx 2.135459$, and we show in Figures 1 (b) and (c) the corresponding plots of $\phi^{*}$ as a function of $d_{N}$.

4.4. The Höfler and Schwarzer (HS) model. Let us now analyze the HS model based on the velocity equation (3.15). This model is the sub-case of (4.7) for $\varphi(z)=\exp z$ and $n \geq 0$ arbitrary. For this model, $\eta_{i}=\eta_{i}^{\prime}$ causes considerable simplification, and the quantities $\gamma_{i}$ given by $(4.10)$ can be expressed as

$$
\gamma_{i}=\phi_{i}(1-\phi)^{n} \eta_{i}\left(\mathcal{S}_{1, i}+\mathcal{S}_{2, i}+\mathcal{S}_{3, i}\right)
$$

where in terms of $\tilde{\eta}_{i}:=\exp \left(\mathbf{s}_{i}^{\mathrm{T}} \Phi\right)$ we define for the HS model

$$
\mathcal{S}_{1, i}:=d_{i}^{2}\left(\tilde{\beta}_{0}+\beta_{1}+\beta_{2}\right)
$$




$$
\begin{aligned}
& \mathcal{S}_{2, i}:=-\sum_{\substack{j=1 \\
j \neq i}}^{N} \frac{\phi_{j} \tilde{\eta}_{j}}{d_{j}^{2} \tilde{\eta}_{j}-d_{i}^{2} \tilde{\eta}_{i}}\left\{\left(d_{i}-d_{j}\right)^{2} \tilde{\beta}_{0}\left(\beta_{1} d_{i} d_{j}+\beta_{2}\left(d_{i}+d_{j}\right)^{2}\right)+\beta_{1} \beta_{2} d_{i} d_{j}\left(d_{i}-d_{j}\right)^{2}\right\}, \\
& \mathcal{S}_{3, i}:=-\tilde{\beta}_{0} \beta_{1} \beta_{2} \sum_{\substack{j, k=1 \\
j<k, j, k \neq i}}^{N} \frac{\phi_{j} \phi_{k} \tilde{n}_{j} \tilde{\eta}_{k} \pi_{i j k}^{2}}{\left(d_{k}^{2} \tilde{\eta}_{k}-d_{i}^{2} \tilde{\eta}_{i}\right)\left(d_{j}^{2} \tilde{\eta}_{j}-d_{i}^{2} \tilde{\eta}_{i}\right)} .
\end{aligned}
$$

Proceeding in a similar way as for the BW model, we now show that $\gamma_{i}<0$ for all vectors $\Phi \in \mathcal{D}_{\phi_{\max }}$, by possibly introducing further structural assumptions on the coefficients $\beta_{0}, \beta_{1}, \beta_{2}$. The decisive difference is, however, that the final result should be valid for the whole range of concentrations from the dilute to the concentrated limit since the HS model is supposed to cover this range, in contrast to the BW model.

The following lemma will be used in slight variants in several instances.

Lemma 4.4. The following inequality holds for $i<j$ :

$$
\frac{\tilde{\eta}_{j}}{d_{i}^{2} \tilde{\eta}_{i}-d_{j}^{2} \tilde{\eta}_{j}} \leq-\left(\mathrm{e}\left(d_{i}^{2}-d_{j}^{2}\right) \sum_{s=1}^{2} \beta_{s}\left(\frac{1}{d_{j}^{s}}-\frac{1}{d_{i}^{s}}\right) \mathbf{d}_{s}^{\mathrm{T}} \Phi\right)^{-1}=-\frac{1}{\mathrm{e}\left(d_{i}^{2}-d_{j}^{2}\right)}\left[\sum_{s=1}^{2} \beta_{s} \frac{d_{i}^{s}-d_{j}^{s}}{d_{i}^{s} d_{j}^{s}} \mathbf{d}_{s}^{\mathrm{T}} \Phi\right]^{-1} .
$$

Proof. We first calculate for $i<j$ :

$$
\begin{aligned}
0 & <\frac{\tilde{\eta}_{j}}{d_{i}^{2} \tilde{\eta}_{i}-d_{j}^{2} \tilde{\eta}_{j}}=\frac{1}{d_{i}^{2} \exp \left(\left(\mathbf{s}_{i}^{\mathrm{T}}-\mathbf{s}_{j}^{\mathrm{T}}\right) \Phi\right)-d_{j}^{2}} \leq \frac{1}{\exp \left(\left(\mathbf{s}_{i}^{\mathrm{T}}-\mathbf{s}_{j}^{\mathrm{T}}\right) \Phi\right)\left(d_{i}^{2}-d_{j}^{2}\right)} \\
& =\frac{\exp \left(\left(\mathbf{s}_{j}^{\mathrm{T}}-\mathbf{s}_{i}^{\mathrm{T}}\right) \Phi\right)}{d_{i}^{2}-d_{j}^{2}}=\exp \left(\sum_{s=1}^{2} \beta_{s}\left(\frac{1}{d_{j}^{s}}-\frac{1}{d_{i}^{s}}\right) \mathbf{d}_{s}^{\mathrm{T}} \Phi\right) \frac{1}{d_{i}^{2}-d_{j}^{2}} .
\end{aligned}
$$

Now, since $d_{i}>d_{j}$ for $i<j$, the argument of the exponential in the last expression is negative. Inequality (4.26) is now a consequence of $\exp (-\alpha) \leq \mathrm{e}^{-1} \alpha^{-1}$ for $\alpha>0$.

The expression (4.26) can be estimated further from above if we drop any of the three summands in the expression in squared brackets. Moreover, we first note that also for this model, $\mathcal{S}_{1, i}<0$. Then we analyze the positive and negative parts of $\mathcal{S}_{2, i}$ and $\mathcal{S}_{3, i}$ separately, and show that we eventually obtain $\gamma_{i}<0$.

Lemma 4.5. Let us rewrite $\mathcal{S}_{2, i}$ as $\mathcal{S}_{2, i}=\mathcal{S}_{2, i}^{+}+\mathcal{S}_{2, i}^{-}$, where $\mathcal{S}_{2, i}^{+}$and $\mathcal{S}_{2, i}^{-}$correspond to the summands of $\mathcal{S}_{2, i}$ with $j>i$ and $j<i$, respectively. Then $\mathcal{S}_{2, i}^{-} \leq 0$, and the following inequality holds:

$$
\mathcal{S}_{2, i}^{+} \leq-\frac{d_{i}^{2}}{\mathrm{e}}\left(\tilde{\beta}_{0}+\beta_{2}\right) \text {. }
$$

Proof. Since $\exp \left(\mathbf{s}_{i}^{\mathrm{T}} \Phi\right)>\exp \left(\mathbf{s}_{j}^{\mathrm{T}} \Phi\right)$ for $i<j$ and $\exp \left(\mathbf{s}_{i}^{\mathrm{T}} \Phi\right)<\exp \left(\mathbf{s}_{j}^{\mathrm{T}} \Phi\right)$ for $i>j$, the factor multiplying $\{\ldots\}$ in the summands of $\mathcal{S}_{2, i}^{-}$is always positive, while $\{\ldots\}<0$. This confirms that $\mathcal{S}_{2, i}^{-} \leq 0$ (note that for $i=1$, the sum is void, i.e. $\left.\mathcal{S}_{2, i}^{-}=0\right)$. To estimate $\mathcal{S}_{2, i}^{+}$, note first that from Lemma 4.4 we may conclude that

$$
\begin{aligned}
\mathcal{S}_{2, i}^{+} & \leq \frac{\tilde{\beta}_{0}}{\mathrm{e}} \sum_{j=i+1}^{N} \frac{\left(\beta_{1} d_{i} d_{j}+\beta_{2}\left(d_{i}+d_{j}\right)^{2}\right)\left(d_{i}-d_{j}\right)^{2} \phi_{j}}{\left(d_{i}^{2}-d_{j}^{2}\right)\left[\beta_{1} \frac{d_{i}-d_{j}}{d_{i} d_{j}} \mathbf{d}_{1}^{\mathrm{T}} \Phi+\beta_{2} \frac{d_{i}^{2}-d_{j}^{2}}{d_{i}^{2} d_{j}^{2}} \mathbf{d}_{2}^{\mathrm{T}} \Phi\right]}-\frac{\beta_{2}}{\mathrm{e}} \sum_{j=i+1}^{N} \frac{\left(d_{i}-d_{j}\right)^{2} d_{i}^{2} d_{j}^{2} \phi_{j}}{\left(d_{i}^{2}-d_{j}^{2}\right)\left(d_{i}-d_{j}\right) \mathbf{d}_{1}^{\mathrm{T}} \Phi} \\
& \leq-\frac{\tilde{\beta}_{0} d_{i}^{2}}{\mathrm{e}} \sum_{j=i+1}^{N} \frac{d_{j}^{2}\left(\beta_{1} d_{i} d_{j}+\beta_{2}\left(d_{i}+d_{j}\right)^{2}\right) \phi_{j}}{\beta_{1} d_{i} d_{j}\left(d_{i}+d_{j}\right) \mathbf{d}_{1}^{\mathrm{T}} \Phi+\beta_{2}\left(d_{i}+d_{j}\right)^{2} \mathbf{d}_{2}^{\mathrm{T}} \Phi}-\frac{d_{i}^{2} \beta_{2}}{\mathrm{e}} \sum_{j=i+1}^{N} \frac{d_{j}^{2} \phi_{j}}{\left(d_{i}+d_{j}\right) \mathbf{d}_{1}^{\mathrm{T}} \Phi} \\
& \leq-\frac{d_{i}^{2} \tilde{\beta}_{0}}{\mathrm{e}} \sum_{j=i+1}^{N} \frac{\left(\beta_{1} d_{i} d_{j}+\beta_{2}\left(d_{i}+d_{j}\right)^{2}\right) d_{j}^{2} \phi_{j}}{\left(\beta_{1} d_{i} d_{j}+\beta_{2}\left(d_{i}+d_{j}\right)^{2}\right) \sum_{k=i+1}^{N} d_{k}^{2} \phi_{k}}-\frac{d_{i}^{2} \beta_{2}}{\mathrm{e}} \sum_{j=i+1}^{N} \frac{d_{j} \phi_{j}}{\mathbf{d}_{1}^{\mathrm{T}} \Phi},
\end{aligned}
$$

which implies (4.27). 
Lemma 4.6. Assume that we rewrite $\mathcal{S}_{3, i}$ as $\mathcal{S}_{3, i}=\mathcal{S}_{3, i}^{-}+\mathcal{S}_{3, i}^{+, 1}+\mathcal{S}_{3, i}^{+, 2}$, where $\mathcal{S}_{3, i}^{+, 1}$ and $\mathcal{S}_{3, i}^{+, 2}$ are the sums over all summands for which $j>i, k>i$ and $k \neq j$ and $j<i, k<i$ and $k \neq j$, respectively. Then we have $\mathcal{S}_{3, i}^{-}<0, \mathcal{S}_{3, i}^{+, 1}>0$ and $\mathcal{S}_{3, i}^{+, 2}>0$. Furthermore, the following inequality holds:

$$
\mathcal{S}_{3, i}^{+, 1} \leq-\frac{d_{i}^{2} \tilde{\beta}_{0}}{\mathrm{e}^{2}} .
$$

Finally, let us assume that the parameters $\boldsymbol{\beta}$ are related to the sizes $\mathbf{d}_{1}$ via the condition

$$
\forall 1 \leq j<i \leq N: \quad \forall \phi \in\left[0, \phi_{\max }\right]: \quad \tilde{H}_{i j}(\phi, \boldsymbol{\beta})<0,
$$

where we define the functions

$$
\tilde{H}_{i j}(\phi, \boldsymbol{\beta}):=-\tilde{\beta}_{0}\left(\beta_{1} d_{i} d_{j}+\beta_{2}\left(d_{i}+d_{j}\right)^{2}\right)-\beta_{2} \beta_{1} d_{i} d_{j}-\phi\left(d_{j}-d_{i}\right)^{2} \tilde{\beta}_{0} \beta_{1} \beta_{2} .
$$

Then

$$
\mathcal{S}_{2, i}^{-}+\mathcal{S}_{3, i}^{+, 2} \leq 0
$$

Proof. The inequalities $\mathcal{S}_{3, i}^{-}<0, \mathcal{S}_{3, i}^{+, 1}>0$ and $\mathcal{S}_{3, i}^{+, 2}>0$ area simple consequence of the fact that only those summands of $\mathcal{S}_{3, i}$ are positive for which either $i<j$ and $i<k$ or $i>j$ and $i>k$, according to the ordering $d_{1}>d_{2}>\cdots>d_{N}$. To deal with

$$
\mathcal{S}_{3, i}^{+, 1}=-\sum_{\substack{j, k=i+1 \\ j<k}}^{N} \frac{\phi_{j} \phi_{k} \tilde{\eta}_{j} \tilde{\eta}_{k} \pi_{i j k}^{2} \tilde{\beta}_{0} \beta_{1} \beta_{2}}{\left(d_{k}^{2} \tilde{\eta}_{k}-d_{i}^{2} \tilde{\eta}_{i}\right)\left(d_{j}^{2} \tilde{\eta}_{k}-d_{i}^{2} \tilde{\eta}_{i}\right)},
$$

note first that, based on formulas similar to (4.26), we get

$$
\begin{aligned}
-\sum_{\substack{j, k=i+1 \\
j<k}}^{N} \frac{\phi_{j} \phi_{k} \tilde{\eta}_{j} \tilde{\eta}_{k} \pi_{i j k}^{2} \tilde{\beta}_{0} \beta_{1} \beta_{2}}{\left(d_{k}^{2} \tilde{\eta}_{k}-d_{i}^{2} \tilde{\eta}_{i}\right)\left(d_{j}^{2} \tilde{\eta}_{j}-d_{i}^{2} \tilde{\eta}_{i}\right)} & \leq-\frac{\tilde{\beta}_{0}}{\mathrm{e}^{2}} \sum_{\substack{j, k=i+1 \\
j \neq k}}^{N} \frac{\phi_{j} \phi_{k} d_{i}^{3} d_{j} d_{k}^{2}\left(d_{i}-d_{j}\right)^{2}\left(d_{j}-d_{k}\right)^{2}\left(d_{k}-d_{i}\right)^{2}}{\left(d_{i}+d_{j}\right)\left(d_{i}-d_{j}\right)^{2}\left(d_{i}+d_{k}\right)^{2}\left(d_{i}-d_{k}\right)^{2} \mathbf{d}_{2}^{\mathrm{T}} \Phi \mathbf{d}_{1}^{\mathrm{T}} \Phi} \\
& \leq-\frac{d_{i}^{2} \tilde{\beta}_{0}}{\mathrm{e}^{2}} \sum_{\substack{j, k=i+1 \\
j \neq k}}^{N} \frac{\phi_{j} \phi_{k} d_{j} d_{k}^{2}}{\mathbf{d}_{2}^{\mathrm{T}} \Phi \mathbf{d}_{1}^{\mathrm{T}} \Phi} \leq-\frac{d_{i}^{2} \tilde{\beta}_{0}}{\mathrm{e}^{2}} .
\end{aligned}
$$

Next, we see that the term

$$
\mathcal{S}_{3, i}^{+, 2}:=-\sum_{\substack{j, k=1 \\ j<k}}^{i-1} \frac{\phi_{j} \phi_{k} \tilde{\eta}_{j} \tilde{\eta}_{k} \pi_{i j k}^{2} \tilde{\beta}_{0} \beta_{1} \beta_{2}}{\left(d_{k}^{2} \tilde{\eta}_{k}-d_{i}^{2} \tilde{\eta}_{i}\right)\left(d_{j}^{2} \tilde{\eta}_{j}-d_{i}^{2} \tilde{\eta}_{i}\right)}
$$

cannot be estimated easily. However, we may "compensate" this term with $\mathcal{S}_{2, i}^{-}$, as expressed in (4.31). (To ensure that our hyperbolicity result is also valid for $N=3, \mathcal{S}_{3, i}^{+, 1}$ should be compensated by one of the terms that have arisen earlier in our analysis.) Observe now that

$$
\mathcal{S}_{2, i}^{-}+\mathcal{S}_{3, i}^{+, 2}=\sum_{j=1}^{i-1} \frac{\phi_{j} \tilde{\eta}_{j}\left(d_{i}-d_{j}\right)^{2}}{d_{j}^{2} \tilde{\eta}_{j}-d_{i}^{2} \tilde{\eta}_{i}} \mathcal{R}_{i j}
$$

where we define

$$
\begin{aligned}
& \mathcal{R}_{i j}:=-\tilde{\beta}_{0}\left(\beta_{1} d_{i} d_{j}+\beta_{2}\left(d_{i}+d_{j}\right)^{2}\right)-\beta_{2} \beta_{1} d_{i} d_{j}+\tilde{\mathcal{R}}_{i j}, \\
& \tilde{\mathcal{R}}_{i j}:=-\tilde{\beta}_{0} \beta_{1} \beta_{2} \sum_{k=j+1}^{i-1} \frac{\phi_{k}\left(d_{k}-d_{i}\right)^{2}\left(d_{k}-d_{j}\right)^{2} \tilde{\eta}_{k}}{d_{k}^{2} \tilde{\eta}_{k}-d_{i}^{2} \tilde{\eta}_{i}} .
\end{aligned}
$$

Since $d_{i}<d_{j}$ and $d_{i}<d_{k}$ in these summands, and the factor multiplying $\mathcal{R}_{i j}$ in (4.32) is positive, we will satisfy (4.31) by achieving that $\mathcal{R}_{i j}<0$. Noting that for $j<k<i$

$$
\frac{\left(d_{k}-d_{i}\right)^{2}\left(d_{k}-d_{j}\right)^{2} \tilde{\eta}_{k}}{d_{k}^{2} \tilde{\eta}_{k}-d_{i}^{2} \tilde{\eta}_{i}}=\frac{\left(d_{k}-d_{i}\right)^{2}\left(d_{k}-d_{j}\right)^{2}}{d_{k}^{2}-d_{i}^{2} \exp \left(\left(\mathbf{s}_{i}^{\mathrm{T}}-\mathbf{s}_{k}^{\mathrm{T}}\right) \Phi\right)} \leq \frac{\left(d_{k}-d_{j}\right)^{2}\left(d_{k}-d_{i}\right)}{d_{k}+d_{i}} \leq\left(d_{j}-d_{i}\right)^{2},
$$


(a)

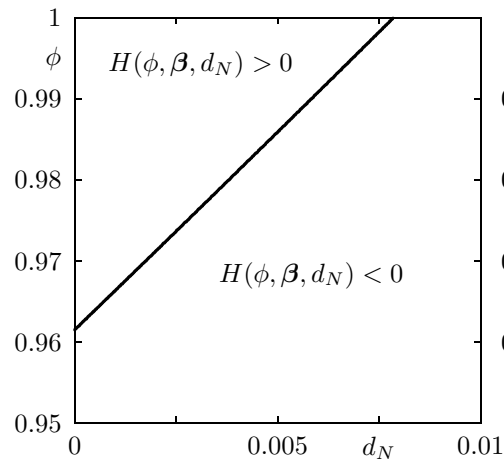

(b)

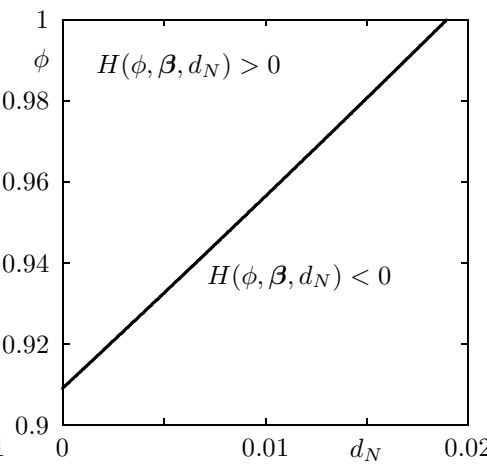

(c)

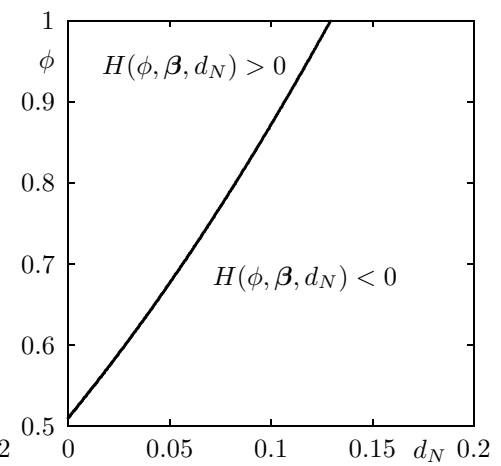

FIG. 2. Region of hyperbolicity $\left(H\left(\phi, \boldsymbol{\beta}, d_{N}\right)<0\right)$ for the HS model (a) for the coefficients (3.13) (with $\beta_{3}=0$ ), (b) for $\beta_{0}=-3.5, \beta_{1}=-1.1, \beta_{2}=-1.02$ (according to (3.12) for large Péclet numbers) and $\beta_{3}=0$, and (c) for $\beta_{0}=-3.42, \beta_{1}=-1.96, \beta_{2}=-1.21$ (according to (3.12) for small Péclet numbers) and $\beta_{3}=0$.

we conclude that $\tilde{\mathcal{R}}_{i j} \leq-\tilde{\beta}_{0} \beta_{1} \beta_{2}\left(d_{j}-d_{i}\right)^{2}\left(\phi_{j+1}+\phi_{j+2}+\cdots+\phi_{i-1}\right)$. Thus, (4.31) holds if the parameters $\boldsymbol{\beta}$ are related to $d_{1}, \ldots, d_{N}$ by $(4.29)$, where $\tilde{H}_{i j}:=\tilde{H}_{i j}(\phi ; \boldsymbol{\beta})$ is defined in (4.30).

Summarizing, and collecting the inequalities for the various terms, we see that if (4.29) is met then

$$
\mathcal{S}_{1, i}+\mathcal{S}_{2, i}+\mathcal{S}_{3, i}=\mathcal{S}_{1, i}+\mathcal{S}_{2, i}^{-}+\mathcal{S}_{2, i}^{+}+\mathcal{S}_{3, i}^{-}+\mathcal{S}_{3, i}^{+, 1}+\mathcal{S}_{3, i}^{+, 2}<\mathcal{S}_{1, i}+\mathcal{S}_{2, i}^{+}+\mathcal{S}_{3, i}^{+, 1} \leq d_{i}^{2} M(\phi, \boldsymbol{\beta})<0,
$$

where we define the function

$$
M(\phi, \boldsymbol{\beta}):=\left(1-\mathrm{e}^{-1}-\mathrm{e}^{-2}\right) \tilde{\beta}_{0}+\beta_{1}+\left(1-\mathrm{e}^{-1}\right) \beta_{2} .
$$

Furthermore, for the discussion of models with a large number of species $N$, the criterion $(4.29)$ with $\tilde{H}_{i j}$ defined by (4.30) is inconvenient, since it requires inspection of a large number of size pairs $d_{i}$ and $d_{j}$. Thus, we propose a sufficient condition for (4.29) to be satisfied for all pairs $j<i$. To this end, we fix a pair $i>j$, define $\delta:=\delta_{i j}=d_{i} / d_{j}$, and divide (4.30) by $d_{j}^{2}$ to obtain

$$
\tilde{H}_{i j}=-\tilde{\beta}_{0}\left(\beta_{1} \delta+\beta_{2}(1+\delta)^{2}\right)-\left(\beta_{2} \beta_{1} \delta\right)-\phi(1-\delta)^{2} \tilde{\beta}_{0} \beta_{1} \beta_{2}
$$

Since $\delta$ varies between $\delta_{N}$ and one, a sufficient condition for (4.29) to be satisfied is given by

$$
\forall \phi \in\left[0, \phi_{\max }\right]: \quad H\left(\phi, \boldsymbol{\beta}, d_{N}\right)<0,
$$

where the following definition of $H\left(\phi, \boldsymbol{\beta}, d_{N}\right)$ is derived from the observation that the two terms in the first line of (4.36) are non-positive, while the term in the second line is non-negative:

$$
H\left(\phi, \boldsymbol{\beta}, d_{N}\right):=-\tilde{\beta}_{0}\left(\beta_{1} d_{N}+\beta_{2}\left(1+d_{N}\right)^{2}\right)-\beta_{2} \beta_{1} d_{N}-\phi\left(1-d_{N}\right)^{2} \tilde{\beta}_{0} \beta_{1} \beta_{2} .
$$

Theorem 4.2. Assume that the vector of parameters $\boldsymbol{\beta}$, the maximum solids concentration $\phi_{\max }$ and the width of the particle size distribution, characterized by the value of $d_{N} \in(0,1]$, are chosen such that the inequality (4.37) is satisfied, where the expression $H\left(\phi, \boldsymbol{\beta}, d_{N}\right)$ is defined by (4.38). Then $\gamma_{i}<0$ for $i=$ $1, \ldots, N$, i.e., the model equations are strictly hyperbolic for $\Phi \in \mathcal{D}_{\phi_{\max }}$.

For the coefficients $\boldsymbol{\beta}$ (with $\beta_{3}=0$ ) given by (3.13) and $n=2$, the curve $H\left(\phi, \boldsymbol{\beta}, d_{N}\right)=0$ is plotted in Figure 2 (a) in a $\phi$ versus $d_{N}$ plot. It turns out that for $d_{N}>d_{N}^{*}:=0.0078595$ (this number is a solution of $\left.H\left(1, \boldsymbol{\beta}, d_{N}\right)=0\right)$, the HS model equations are strictly hyperbolic in $\mathcal{D}_{\phi_{\max }}$ without any restrictions on $\phi_{\max }$. Note that for $0<d_{N}<d_{N}^{*}$ smaller than that, condition (4.37) is violated only for values of $\phi_{\max }$ very close to one. In fact, Figure 2 (a) indicates that the HS model with the parameters (3.13) is strictly hyperbolic for arbitrarily small values of $d_{N}$ if we set $\phi_{\max } \leq 0.96$. Given that $d_{N}^{*}$ is already a small number, we can say that hyperbolicity holds for almost all cases of practical interest for this model. 

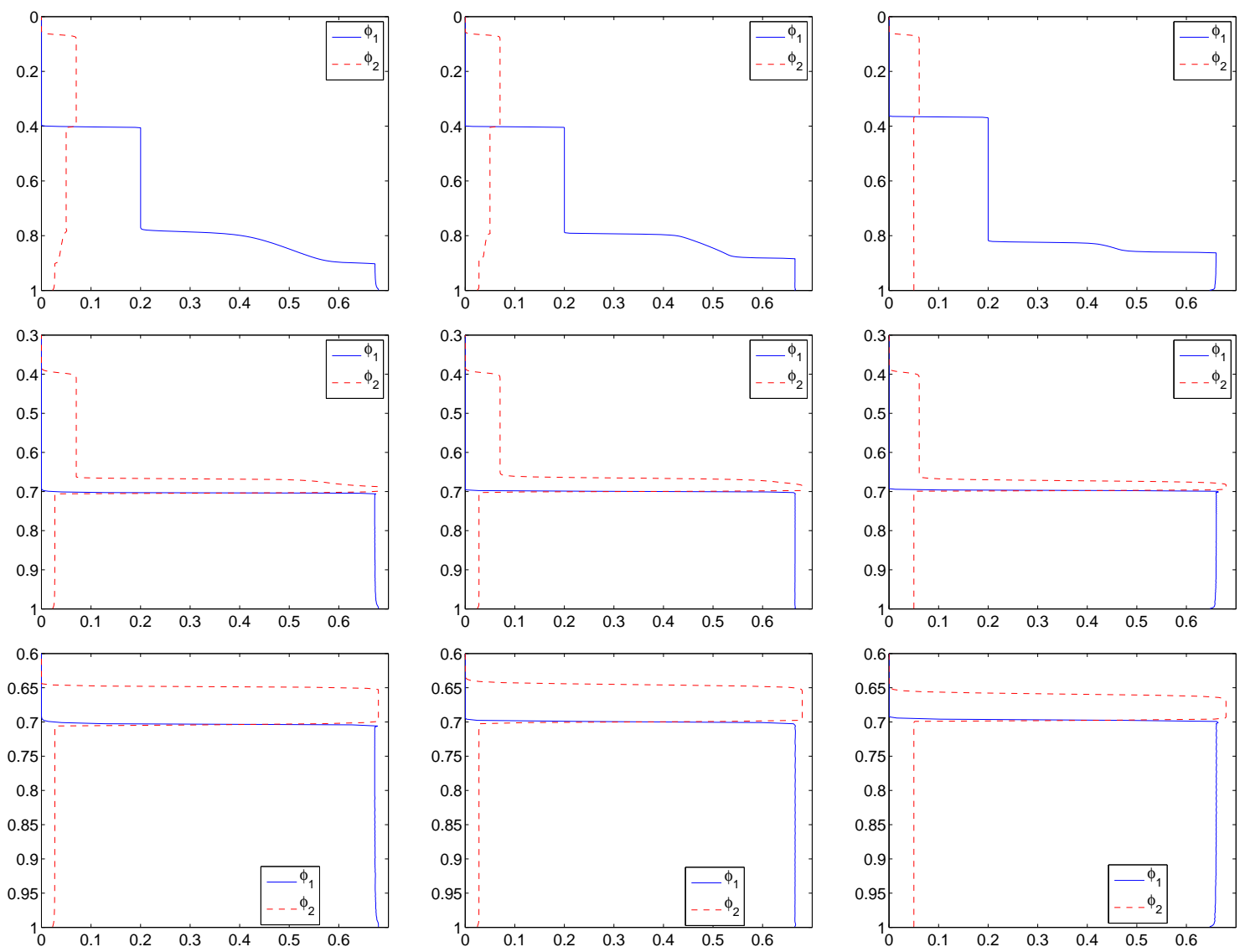

FIG. 3. Example 1 (MLB and HS models with $N=2$ ): numerical solution at $t=50 \mathrm{~s}$ (top), $t=300 \mathrm{~s}$ (middle) and $t=1000 \mathrm{~s}$ (bottom). Here and in Figures 4 and 5 , the left and middle column correspond to the Roe and KT scheme, respectively, applied to the MLB model and the right column corresponds to the KT scheme applied to the HS model; the horizontal axis in each plot denotes concentration, and the vertical axis denotes normalized depth.

As for the BW model, we also illustrate the result obtained for the coefficients $\boldsymbol{\beta}$ given (3.12) with the respective values of $\beta_{3}$ by zero. Figures 2 (b) and (c) show the corresponding regions of guaranteed hyperbolicity for these cases.

Finally, we remark here that the value $n=2$ in (3.15) was utilized in the examples of Figure 2, and that very similar curves are obtained for alternative values $1 \leq n \leq 5$.

\section{NUMERICAL EXAMPLES}

We apply the Roe scheme and KT schemes to the MLB and HS models to simulate batch settling of a suspension with equal-density particles in a vessel of normalized depth one. We first briefly describe both schemes, and refer to [32] and [31] for the Roe and KT scheme, respectively. We discretize the spatial domain $[0,1]$ into $M$ cells of size $\Delta x=1 / M$. The time step is denoted by $\Delta t$, and we define $x_{j}:=j \Delta x$ and $t_{n}:=n \Delta t$. Furthermore, we assume that $\lambda:=\Delta t / \Delta x$ is fixed by an appropriate CFL condition.

5.1. The Roe and KT schemes. The conservative form of the Roe scheme for (1.1) is given by

$$
\Phi_{j}^{n+1}=\Phi_{j}^{n}-\lambda\left(\mathcal{F}_{j+1 / 2}^{n}-\mathcal{F}_{j-1 / 2}^{n}\right), \quad j=1, \ldots, M, \quad n=0,1,2, \ldots,
$$



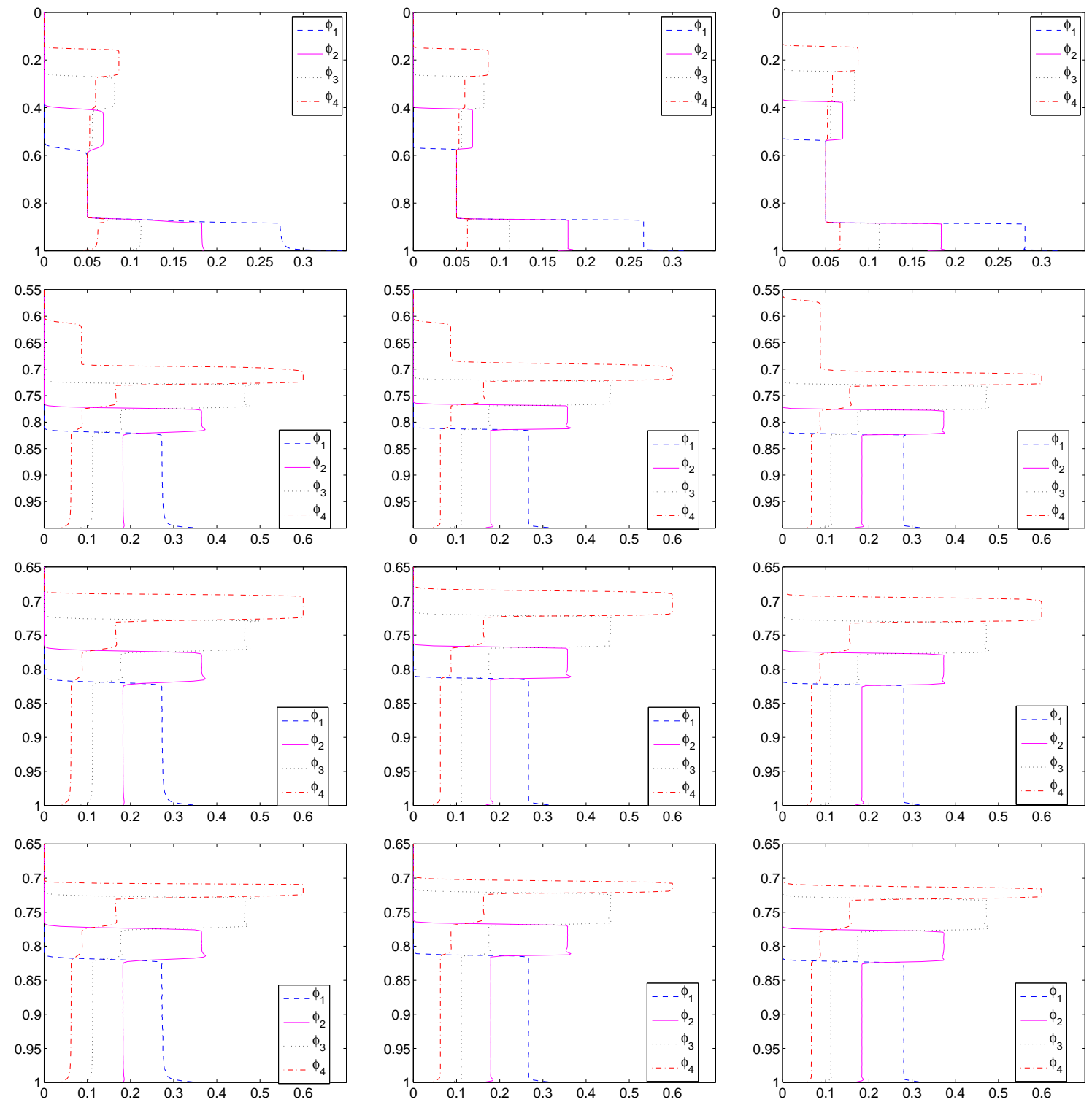

Fig. 4. Example 2 (MLB and HS models, $N=4$ ): numerical solution at $t=50 \mathrm{~s}$ (top row), $t=200 \mathrm{~s}$ (second row), $t=300 \mathrm{~s}$ (third row) and $t=1000 \mathrm{~s}$ (bottom row) with $\Delta x=0.0005$.

where $\Phi_{j}^{n}=\left(\phi_{1, j}^{n}, \ldots, \phi_{N, j}^{n}\right)^{\mathrm{T}}$ and the numerical flux vector is defined as

$$
\mathcal{F}_{j+1 / 2}^{n}=\frac{1}{2}\left[\mathbf{f}\left(\Phi_{j+1}^{n}\right)+\mathbf{f}\left(\Phi_{j}^{n}\right)\right]-\frac{1}{2}\left(\alpha_{1}\left|\lambda_{1}\right| \mathbf{r}_{1}+\cdots+\alpha_{N}\left|\lambda_{N}\right| \mathbf{r}_{N}\right) .
$$

Here, $\lambda_{1}, \ldots, \lambda_{N}$ are the eigenvalues of the Jacobian $\mathcal{J}_{\mathrm{f}}$ evaluated at $\Phi_{j+1 / 2}^{n}=\frac{1}{2}\left(\Phi_{j+1}^{n}+\Phi_{j}^{n}\right)$, which are calculated by a root finder starting from the velocities $v_{1}\left(\Phi_{j+1 / 2}^{n}\right), \ldots, v_{N}\left(\Phi_{j+1 / 2}^{n}\right)$. The components of $\boldsymbol{\alpha}=\left(\alpha_{1}, \ldots, \alpha_{N}\right)^{\mathrm{T}}$ are defined by $\Phi_{j+1}^{n}-\Phi_{j}^{n}=\alpha_{1} \mathbf{r}_{1}+\cdots+\alpha_{N} \mathbf{r}_{N}$, or equivalently, $\boldsymbol{\alpha}=\mathbf{R}^{-1}\left(\Phi_{j+1}^{n}-\Phi_{j}^{n}\right)$. Here, $\mathbf{r}_{1}, \ldots, \mathbf{r}_{N}$ are the normalized right eigenvectors of $\mathcal{J}_{\mathbf{f}}\left(\Phi_{j+1 / 2}^{n}\right)$, which form the columns of $\mathbf{R}$. The characteristic information is given by the secular equation and (4.2) and (4.3) for the MLB model. For a 


\begin{tabular}{lccccccccccc}
\hline$i$ & 1 & 2 & 3 & 4 & 5 & 6 & 7 & 8 & 9 & 10 & 11 \\
\hline$\phi_{i}^{0}\left[10^{-3}\right]$ & 0.435 & 3.747 & 14.420 & 32.603 & 47.912 & 47.762 & 32.663 & 15.104 & 4.511 & 0.783 & 0.060 \\
$D_{i}\left[10^{-5} \mathrm{~m}\right]$ & 8.769 & 8.345 & 7.921 & 7.497 & 7.073 & 6.649 & 6.225 & 5.801 & 5.377 & 4.953 & 4.529 \\
$d_{i}$ & 1.000 & 0.952 & 0.903 & 0.855 & 0.807 & 0.758 & 0.710 & 0.662 & 0.613 & 0.565 & 0.516 \\
\hline
\end{tabular}

TABLE 1. Example 3 (MLB and HS models, $N=11$ ): initial concentrations $\phi_{i}^{0}$, real particle sizes $D_{i}$, and normalized particle sizes $d_{i}$.

given CFL number CFL, we employ $\Delta t=\operatorname{CFL} \Delta x / \rho$, where $\rho$ the biggest characteristic velocity, i.e.,

$$
\rho=\max _{j=1, \ldots, M} \max _{i=1, \ldots, N}\left|\lambda_{i}\left(\Phi_{j+1 / 2}\right)\right|
$$

In contrast to the just-described Roe scheme, the second-order central KT scheme [31] does not require knowledge of the complete eigenstructure of the problem. However, this method does rely on the local speed of wave propagation. The semi-discrete conservative form of KT scheme is

$$
\frac{d \Phi_{j}}{d t}=-\frac{1}{\Delta x}\left(\mathcal{H}_{j+1 / 2}-\mathcal{H}_{j-1 / 2}\right)
$$

with the numerical flux vector

$$
\mathcal{H}_{j+1 / 2}:=\frac{1}{2}\left[\mathbf{f}\left(\Phi_{j+1 / 2}^{+}\right)-\mathbf{f}\left(\Phi_{j+1 / 2}^{-}\right)\right]-\frac{a_{j+1 / 2}}{2}\left(\Phi_{j+1 / 2}^{+}-\Phi_{j+1 / 2}^{-}\right) .
$$

The extrapolated values $\Phi_{j+1 / 2}^{ \pm}$are $\Phi_{j+1 / 2}^{+}=\Phi_{j+1}-(\Delta x / 2)\left(\Phi_{x}\right)_{j+1}$ and $\Phi_{j+1 / 2}^{-}=\Phi_{j}+(\Delta x / 2)\left(\Phi_{x}\right)_{j}$, where $a_{j+1 / 2}$ is the maximal local speed, which we take as

$$
a_{j+1 / 2}:=\max \left\{\rho\left(\mathcal{J}_{\mathbf{f}}\left(\Phi_{j+1 / 2}^{+}\right)\right), \rho\left(\mathcal{J}_{\mathbf{f}}\left(\Phi_{j+1 / 2}^{-}\right)\right)\right\},
$$

where $\rho\left(\mathcal{J}_{\mathbf{f}}(\cdot)\right)$ denotes the spectral radius of the matrix $\mathcal{J}_{\mathbf{f}}(\cdot)$. The approximate spatial derivative of $\Phi(x, t)$ is computed using a $\theta$-minmod limiter for each component of $\Phi$. In our implementations, we use $\theta=1.3$.

For time discretization we use the optimal third-order TVD Runge-Kutta methods (see e.g. [44]) with with CFL $=0.5$. Then we take $\Delta t=\mathrm{CFL} \Delta x / S_{\max }^{n}$, where $S_{\max }^{n}$ denotes the biggest local propagation speed throughout the domain at time $t_{n}$. For both the Roe and KT schemes we utilize zero-flux boundary conditions, i.e. $\mathbf{f}_{x=0}=\mathbf{f}_{x=L}=0$, which in the numerical approach corresponds to take numerical fluxes equal to zero at both ends of the spatial domain.

5.2. Numerical examples. The numerical experiments are similar to those of [17]; Examples 1, 2 and 3 correspond to the cases $N=2, N=4$ and $N=11$, respectively. All examples are based on the physical parameters $g=9.81 \mathrm{~m} / \mathrm{s}^{2}, \mu_{\mathrm{f}}=0.02416 \mathrm{Pas}, \varrho_{\mathrm{f}}=1208 \mathrm{~kg} / \mathrm{m}^{3}, \varrho_{1}=\cdots=\varrho_{N}=\varrho_{\mathrm{s}}=2790 \mathrm{~kg} / \mathrm{m}^{3}$ that correspond to a standard published experiment [41]. The function $V(\phi)$ in the MLB model has the exponent $n=4.7$, except for $N=11$ in which case we choose $n=4.65$, while the parameters $\beta_{0}, \ldots, \beta_{3}$ for the HS models are those given by (3.13) (with $\beta_{3}=0$ ).

In Example 1, the original depth of the vessel is $L=0.3 \mathrm{~m}$; this is also true for $N=4$, and the unnormalized particle diameters are $D_{1}=4.96 \times 10^{-4} \mathrm{~m}$ and $D_{2}=1.25 \times 10^{-4} \mathrm{~m}$, corresponding to $d_{1}=1$ and $d_{2}=D_{2} / D_{1}=0.25202$. The maximum total concentration is $\phi_{\max }=0.68$, along with the initial concentrations $\Phi^{0}=\left(\phi_{1}^{0}, \phi_{2}^{0}\right)=(0.2,0.05)^{\mathrm{T}}$. For Example 2, we choose $d_{1}=1, d_{2}=0.8, d_{3}=0.6$ and $d_{4}=0.4, \phi_{\max }=0.6$, and $\phi_{i}^{0}=0.05$ for $i=1, \ldots, 4$. Finally, in Example 3 for $N=11$, which is based on experimental data from [43], we consider $L=0.935 \mathrm{~m}$ and $\phi_{\max }=0.641$. The initial concentrations $\phi_{i}^{0}$, diameters $D_{i}$ and normalized diameters $d_{i}=D_{i} / D_{1}$ are given in Table 1.

\section{Conclusions}

Our analysis illustrates the use of the secular equation as a tool for the hyperbolicity analysis for polydisperse sedimentation models, and leads to estimates of hyperbolicity regions that qualitatively agree with the ranges of validity of the MLB, BW and HS models; recall that the BW model is valid for dilute suspensions only, which is consistent with the limitations visible in Figure 1. For the BW and HS models, only the sign 

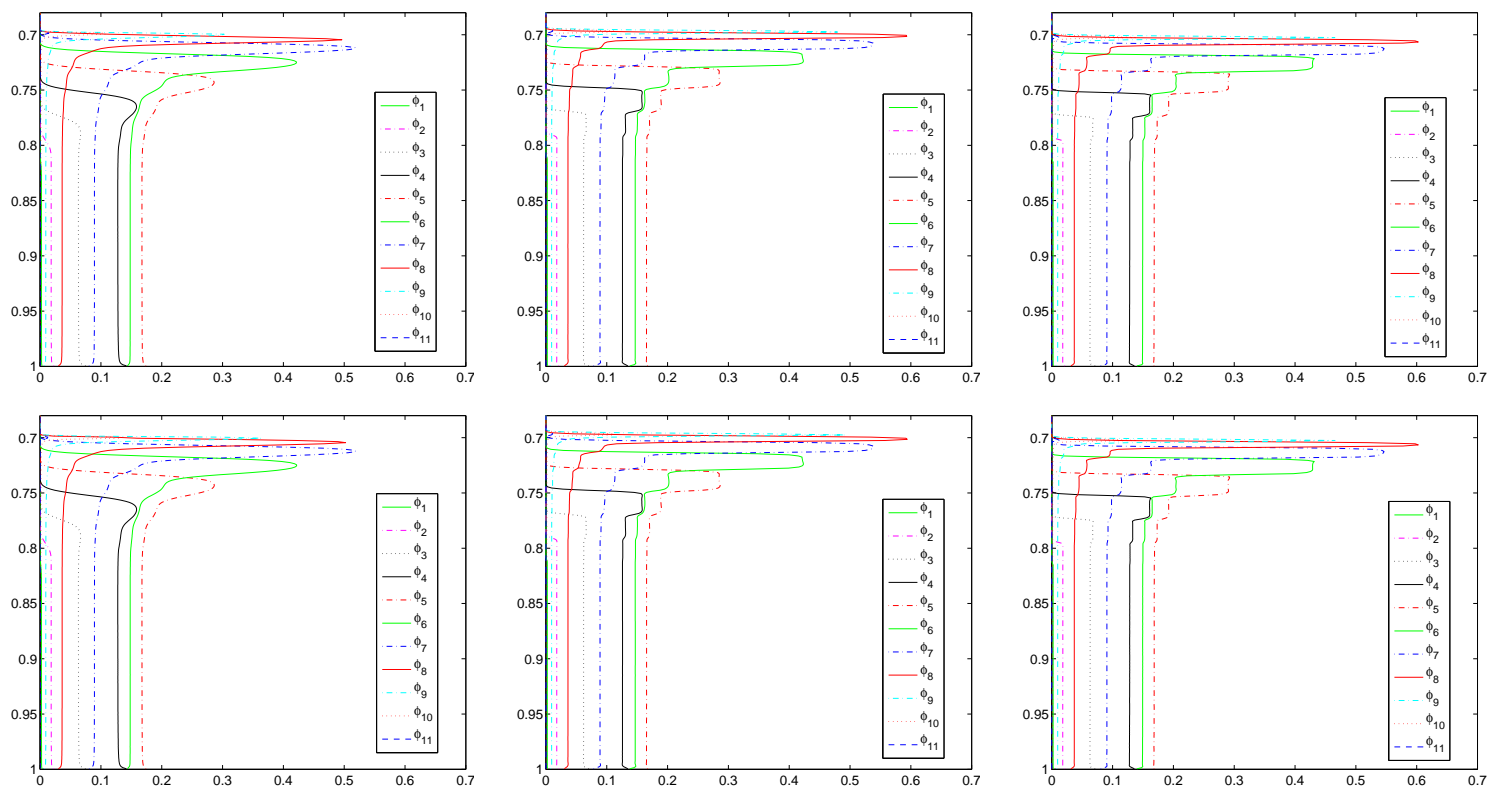

FIG. 5. Example 3 (MLB and HS models, $N=11$ ): numerical solution at $t=600 \mathrm{~s}$ (top) and $t=1000 \mathrm{~s}$ (bottom) with $\Delta x=0.0005$.

of the coefficients $\beta_{0}, \ldots, \beta_{3}$, but not the values enter our analysis; results will only change quantitatively for other sets of parameters. In addition, a similar analysis could be advanced for the case that $\beta_{3}$ is a small, but positive parameter. Previous works [23, 24, 52] already illustrated applications of the secular equation [1] for perturbations of diagonal matrices of ranks $m=1$ and $m=2$ (for the multi-class Lighthill-WhithamRichards traffic and the MLB sedimentation model, respectively), but it is here for the first time (to our knowledge) that this result is led to practical use for $m=3$.

Clearly, our analysis is subject to limitations in terms of the accuracy of the estimates of the hyperbolicity region. Theorems 4.1 and 4.2 state in which regions hyperbolicity is ensured, that is, where we can guarantee that $\gamma_{i} \cdot \gamma_{j}>0$. However, this property is a sufficient, but not a necessary condition to ensure hyperbolicty; the models may well be hyperbolic in other sub-regions of parameter space, but with $\gamma_{i} \cdot \gamma_{j} \leq 0$ for some choices of $i$ and $j$. While this is an intrinsic limitation of the secular equation, our analysis of the HS model shows that slightly larger hyperbolicity regions may be obtained for a given set of particle sizes $d_{1}, \ldots, d_{N}$ if the functions $\tilde{H}_{i j}$ given by (4.36) (rather than the single function $H\left(\phi, \boldsymbol{\beta}, d_{N}\right)$ ) are evaluated. Also, in realistic models the phase space is not simply limited by a hyperplane $\phi=\phi_{\max }$, but by a so-called dense packing manifold, which is a curved surface in $\overline{\mathcal{D}}_{1}$.

Despite these limitations, the present calculus can be extended in several possible ways. First of all, we selected the MLB, BW and HS models because the computations are slightly different in each of these cases. In particular, our interest in the HS model is motivated by a result from [14] stating that the HS model is, unlike the DG model, hyperbolic for $N=2$ without further restrictions. More advanced models that should be analyzed include the models presented in [28, 36, 42]. The model by Patwardhan and Tien [36] generalizes the MLB model, and utilizes a more involved for $v_{i}$. The models in [28] are further modifications of the BW model, they consider $S_{i j}$ to be a rational (rather than polynomial) function of $d_{j} / d_{i}$. Finally, the difficulty associated with the model by Selim et al. [42], which is otherwise similar to the MLB model, consists in the postulated dependence of $v_{i}$ on partial sums like $\phi_{1}+\cdots+\phi_{i-1}$; it is unclear at the moment whether this model can be transformed so that (1.2) is satisfied.

Let us also mention that although we focus here on spatially one-dimensional models, the present hyperbolicity calculus remains valid for the two- or three-dimensional version of (1.1). In fact, in that case 
the model equation (1.1) is replaced by $\partial_{t} \phi_{i}+\nabla \cdot\left(\mathbf{q} \phi_{i}+f_{i}(\Phi) \mathbf{k}\right)=0$ for $i=1, \ldots, N$, where $\mathbf{q}$ is the volume-averaged mixture flow velocity (for which additional equations, e.g. a version of the Navier-Stokes equations have to be solved), and $\mathbf{k}$ is the downwards-pointing unit vector. This equation is hyperbolic if and only if (1.1) with the same vector $\mathbf{f}(\Phi)=\left(f_{1}(\Phi), \ldots, f_{N}(\Phi)\right)^{\mathrm{T}}$ is (see [11] for details).

Concerning the numerical examples, observe that in all examples the model parameters have been chosen such that both the MLB and HS models are strictly hyperbolic on $\mathcal{D}_{\phi_{\max }}$ according to the analysis of Section 4. Our results illustrate that for both the MLB and HS models, solutions for equal-density spheres with a constant initial composition $\Phi^{0}$ typically evolve as follows: if $\Phi^{0} \in \mathcal{D}_{\phi_{\max }}$, i.e., $\phi_{i}^{0}>0$ for $i=1, \ldots, N$, then the bottommost zone will contain particles of all species, the next zone will contain species 2 to $N$ (i.e., $\phi_{1}=0$ ), the next only species 3 to $N$ (i.e., $\phi_{1}=\phi_{2}=0$ ), and so on, until a zone is reached in which only (the smallest) species $N$ is present, followed by a zone void of particles $(\Phi=0)$. The composition of each of these zones corresponds to the situation addressed in Remark 2.2, i.e., strict hyperbolicity is ensured, and is also obtained from a method of solution based on the construction of kinematic shocks that separate areas of constant composition [27, 41].

Having said this, we mention that the construction of exact solutions to the system of conservation laws (1.1) that satisfy an entropy condition is at least complicated since most choices of $\mathbf{f}(\Phi)$ will lead to a system of conservation laws that in the best case is provably strictly hyperbolic on $\mathcal{D}_{\phi_{\max }}$ (as a consequence of our analysis), but whose characteristic fields in general neither linearly degenerate nor genuinely nonlinear, which rules out, for example, the use of Lax's shock admissibility criterion. A suitable shock admissibility criterion is Liu's entropy condition (see e.g. [20] for details on these criteria). The construction of solutions that satisfy this condition has been undertaken so far only for $N=2[9]$.

Moreover, we have chosen our numerical simulations fairly simple schemes, which nevertheless utilize characteristic information that we do now have access to thanks to the secular equation. However, the true strength of the availability of characteristic information lies in the possibility to utilize high-resolution "spectral" schemes, such as the one introduced in [24] for the multiclass LWR traffic model. In terms of resolution these schemes are a potential serious alternative to component-wise discretizations such as WENO [51] or WENO-multiresolution [17] schemes, which are the standard at present since the effort needed to obtain this information has hitherto been considered excessive. We will come back to spectral schemes for the present models in a forthcoming paper.

\section{ACKNOWLEDGMENTS}

R. Bürger acknowledges partial support by Fondecyt project 1090456, Fondap in Applied Mathematics, project 15000001, BASAL project CMM, Universidad de Chile and Centro de Investigación en Ingeniería Matemática ( $\left.\mathrm{CI}^{2} \mathrm{MA}\right)$, Universidad de Concepción, and project AMIRA P996/INNOVA 08CM01-17 "Instrumentación y Control de Espesadores". P. Mulet and R. Donat are partially supported by Spanish MCINN MTM2008-00974.

\section{REFERENCES}

[1] J. Anderson. A secular equation for the eigenvalues of a diagonal matrix perturbation. Lin. Alg. Appl., 246:49-70, 1996.

[2] M. Bargieł and E.M. Tory. Stability of tridisperse suspensions. Comput. Vis. Sci., 10:163-170, 2007.

[3] D.K. Basson, S. Berres, and R. Bürger. On models of polydisperse sedimentation with particle-size-specific hindered-settling factors. Appl. Math. Modelling, 33:1815-1835, 2009.

[4] G.K. Batchelor. Sedimentation in a dilute polydisperse system of interacting spheres. Part 1. General theory. J. Fluid Mech., 119:379-408, 1982.

[5] G.K. Batchelor and R.W. Janse van Rensburg. Structure formation in bidisperse sedimentation. J. Fluid Mech., 166:379407, 1986

[6] G.K. Batchelor and C.S. Wen. Sedimentation in a dilute polydisperse system of interacting spheres. Part 2. Numerical results. J. Fluid Mech., 124:495-528, 1982.

[7] S. Benzoni-Gavage and R.M. Colombo. An n-populations model for traffic flow. Eur. J. Appl. Math., 14:587-612, 2003.

[8] S. Berres and R. Bürger. On the settling of a bidisperse suspension with particles having different sizes and densities. Acta Mech., 201:47-62, 2008.

[9] S. Berres and R. Bürger. On Riemann problems and front tracking for a model of sedimentation of polydisperse suspensions. ZAMM Z. Angew. Math. Mech., 87:665-691, 2007. 
BÜRGER, DONAT, MULET, AND VEGA

[10] S. Berres, R. Bürger, and K.H. Karlsen. Central schemes and systems of conservation laws with discontinuous coefficients modeling gravity separation of polydisperse suspensions. J. Comput. Appl. Math., 164-165:53-80, 2004.

[11] S. Berres, R. Bürger, K.H. Karlsen, and E.M. Tory. Strongly degenerate parabolic-hyperbolic systems modeling polydisperse sedimentation with compression. SIAM J. Appl. Math., 64:41-80, 2003.

[12] P.M. Biesheuvel, H. Verweij, and V. Breedveld. Evaluation of instability criterion for bidisperse sedimentation. AIChE J., 47:45-52, 2001

[13] R. Bürger, K.-K. Fjelde, K. Höfler, and K.H. Karlsen. Central difference solutions of the kinematic model of settling of polydisperse suspensions and three-dimensional particle-scale simulations. J. Engrg. Math., 41:167-187, 2001.

[14] R. Bürger, K.H. Karlsen, E.M. Tory, and W.L. Wendland. Model equations and instability regions for the sedimentation of polydisperse suspensions of spheres. ZAMM Z. Angew. Math. Mech., 82:699-722, 2002.

[15] R. Bürger, A. García, K.H. Karlsen, and J.D. Towers. A family of schemes for kinematic flows with discontinuous flux. J. Engrg. Math., 60:387-425, 2008.

[16] R. Bürger, A. García, and M. Kunik. A generalized kinetic model of sedimentation of polydisperse suspensions with a continuous particle size distribution. Math. Models Methods Appl. Sci., 18:1741-1785, 2008.

[17] R. Bürger and A. Kozakevicius. Adaptive multiresolution WENO schemes for multi-species kinematic flow models. J. Comput. Phys., 224:1190-1222, 2007.

[18] A. Cauchy. Mécanique céleste. Sur le mouvement de notre système planetaire. In: Cuvres Complètes d'Augustin Cauchy, Ière Sér., Gauthier-Villars, Paris, vol. 5, no. 98, 331-341, 1885.

[19] A. Cuthbertson, P. Dong, S. King, and P. Davies. Hindered settling velocity of cohesive/non-cohesive sediment mixtures. Coastal Engrg., 55:1197-1208, 2008.

[20] C.M. Dafermos, Hyperbolic Conservation Laws in Continuum Physics, Second Ed., Springer Verlag, Berlin, 2005.

[21] R.H. Davis and H. Gecol. Hindered settling function with no empirical parameters for polydisperse suspensions. AIChE J., 40:570-575, 1994.

[22] R.H. Davis and H. Gecol. Classification of concentrated suspensions using inclined settlers. Int. J. Multiphase Flow, 22:563$574,1996$.

[23] R. Donat and P. Mulet. A secular equation for the Jacobian matrix of certain multi-species kinematic flow models. Numer. Methods Partial Differential Equations, to appear.

[24] R. Donat and P. Mulet. Characteristic-based schemes for multi-class Lighthill-Whitham-Richards traffic models. J. Sci. Comput., 37:233-250, 2008.

[25] G.H. Golub. Some modified matrix eigenvalue problems. SIAM Review, 15:318-334, 1973.

[26] M. Gray, Z. Xu, and J. Masliyah. Physics in the oil sands of Alberta. Physics Today, 62(3):31-35, 2009.

[27] H.P. Greenspan and M. Ungarish. On hindered settling of particles of different sizes. Int. J. Mutiphase Flow, 8:587-604, 1982.

[28] Z. Ha and S. Liu. Settling velocities of polydisperse concentrated suspensions. Canad. J. Chem. Engrg., 80:783-790, 2002.

[29] K. Höfler, Simulation and modeling of mono- and bidisperse suspensions. Doctoral Thesis, Institut für Computeranwendungen, Universität Stuttgart, Germany, 2000.

[30] K. Höfler and S. Schwarzer. The structure of bidisperse suspensions al low Reynolds numbers. In A.M. Sändig, W. Schiehlen and W.L. Wendland (eds.), Multifield Problems: State of the Art, Springer Verlag, Berlin, 42-49, 2000.

[31] A. Kurganov and E. Tadmor. New high-resolution central schemes for nonlinear conservation laws and convection-diffusion equations. J. Comput. Phys., 160:241-282, 2000.

[32] R. J. LeVeque. Finite Volume Methods for Hyperbolic Problems. Cambridge University Press, Cambridge, UK, 2002.

[33] M.J. Lockett and K.S. Bassoon. Sedimentation of binary particle mixtures. Powder Technol., 24:1-7, 1979.

[34] J.H. Masliyah. Hindered settling in a multiple-species particle system. Chem. Engrg. Sci., 34:1166-1168, 1979.

[35] H. Nasr-el-Din, J.H. Masliyah, and K. Nandakumar. Continuous separation of suspensions containing light and heavy particle species. Canad. J. Chem. Engrg. 77:1003-1012, 1999.

[36] V.S. Patwardhan and C. Tien. Sedimentation and liquid fluidization of solid particles of different sizes and densities. Chem. Engrg. Sci., 40:1051-1060, 1985.

[37] S. Qian, R. Bürger, and H.H. Bau. Analysis of sedimentation biodetectors. Chem. Engrg. Sci. 60:2585-2598, 2005.

[38] J.F. Richardson and W.N. Zaki. Sedimentation and fluidization: Part I. Trans. Instn. Chem. Engrs. (London), 32:35-53, 1954.

[39] F. Rosso and G. Sona. Gravity-driven separation of oil-water dispersions. Adv. Math. Sci. Appl., 11:127-151, 2001.

[40] W.B. Russel, D.A. Saville, and W.R. Schowalter. Colloidal Dispersions. Cambridge University Press, Cambridge, UK, 1989.

[41] W. Schneider, G. Anestis, and U. Schaflinger. Sediment composition due to settling of particles of different sizes. Int. J. Multiphase Flow 11:419-423, 1985.

[42] M.S. Selim, A.C. Kothari, and R.M. Turian. Sedimentation of multisized particles in concentrated suspensions. AIChE J., 29:1029-1038, 1983.

[43] P.T. Shannon, E. Stroupe, and E.M. Tory. Batch and continuous thickening. Ind. Engrg. Chem. Fund., 2:203-211, 1963.

[44] C.-W. Shu. Essentially non-oscillatory and weighted essentially non-oscillatory schemes for hyperbolic conservation laws. In B. Cockburn, C. Johnson, C.W. Shu, E. Tadmor, A. Quarteroni, editors, Advanced Numerical Approximation of Nonlinear Hyperbolic Equations, Lecture Notes in Mathematics, pp 325-432. Springer, New York, 1998. 
[45] R. Simura and K. Ozawa. Mechanism of crystal redistribution in a sheet-like magma body: Constraints from the Nosappumisaki and other Shoshonite intrusions in the Nemuro prenisula, Northern Japan. J. Petrology, 47:1809-1851, 2006.

[46] R.H. Weiland, Y.P. Fessas, and B.V. Ramarao. On instabilities arising during sedimentation of two-component mixtures of solids. J. Fluid Mech., 142:383-389, 1984.

[47] C.S. Wen. The Fundamentals of Aerosol Dynamics. World Scientific, Singapore, 1996.

[48] G.C.K. Wong and S.C.K. Wong. A multi-class traffic flow model-an extension of LWR model with heterogeneous drivers. Transp. Res. A, 36:827-841, 2002.

[49] B. Xue and Y. Sun. Modeling of sedimentation of polydisperse spherical beads with a broad size distribution. Chem. Engrg. Sci., 58:1531-1543, 2003.

[50] A. Zeidan, S. Rohani, and A. Bassi. Dynamic and steady-state sedimentation of polydisperse suspension and prediction of outlets particle-size distribution. Chem. Engrg. Sci., 59:2619-2632, 2004.

[51] P. Zhang, S.C. Wong, and C.-W. Shu, A weighted essentially non-oscillatory numerical scheme for a multi-class traffic flow model on an inhomogeneous highway. J. Comput. Phys., 212:739-756, 2006.

[52] P. Zhang, R.X. Liu, S.C. Wong, and S.Q. Dai. Hyperbolicity and kinematic waves of a class of multi-population partial differential equations. Eur. J. Appl. Math., 17:171-200, 2006. 